Canad. J. Math. Vol. 71 (3), 2019 pp. 533-555

http://dx.doi.org/10.4153/CJM-2017-038-8

() Canadian Mathematical Society 2018

\title{
Lipschitz 1-connectedness for Some Solvable Lie Groups
}

\author{
David Bruce Cohen
}

\begin{abstract}
A space $X$ is said to be Lipschitz 1-connected if every Lipschitz loop $\gamma: S^{1} \rightarrow X$ bounds a $O(\operatorname{Lip}(\gamma))$-Lipschitz disk $f: D^{2} \rightarrow X$. A Lipschitz 1-connected space admits a quadratic isoperimetric inequality, but it is unknown whether the converse is true. Cornulier and Tessera showed that certain solvable Lie groups have quadratic isoperimetric inequalities, and we extend their result to show that these groups are Lipschitz 1-connected.
\end{abstract}

\section{Introduction}

\subsection{Lipschitz 1-connectedness}

Consider a complete, simply connected Riemannian manifold $X$ that is homogeneous in the sense that some Lie group acts on $X$ transitively by isometries. It is well known that in such a manifold, every loop admits a Lipschitz filling (see Proposition 2.6; for a much stronger result see [9, Corollary 1.4]). A classical way to study the large scale geometry of $X$ is by asking how hard it is to fill loops in $X$.

Definition 1.1 Let $\gamma: S^{1} \rightarrow X$ be a loop. The filling span of $\gamma$, denoted $\operatorname{Span}(\gamma)$, is defined to be $\inf \left\{\operatorname{Lip}(f)\left|f: D^{2} \rightarrow X ; f\right|_{S^{1}}=\gamma\right\}$.

We say that $X$ is Lipschitz 1-connected if there exists a constant $C$ such that

$$
\operatorname{Span}(\gamma) \leq C \operatorname{Lip}(\gamma)
$$

for all Lipschitz loops $\gamma: S^{1} \rightarrow X$. (The term filling span is derived from Gromov's filling span function $[6, \S 5])$.

For instance, Euclidean $n$-dimensional space $\mathbb{E}^{n}$ is Lipschitz 1-connected because one may "cone off" loops in $\mathbb{E}^{n}$ : given a loop $\gamma: S^{1} \rightarrow X$ with $\gamma(1)=0$, we may take $f\left(r e^{i \theta}\right)=r \gamma\left(e^{i \theta}\right)$ to obtain a 1-Lipschitz filling. A similar argument shows that all CAT(0) manifolds are Lipschitz 1-connected.

\subsection{Solvable Groups}

In this paper, we will be interested in the case where $X$ is some solvable real Lie group $G$ equipped with a left invariant metric. To motivate this, note that Lipschitz 1-connectedness is a QI-invariant (Proposition 2.7), and that every Lie group is quasi

Received by the editors August 7, 2017.

Published electronically April 24, 2018.

This work was supported by NSF award 1148609.

AMS subject classification: 20F65, 22E25.

Keywords: Dehn function, solvable group, Lipschitz 1-connectedness. 
isometric to a solvable Lie group. We will further assume that $G$ has the form $U \rtimes A$, where the following conditions hold.

- $A$ is an abelian Lie group.

- $U$ is a closed subgroup of the group of real $n \times n$ upper triangular matrices with diagonal entries equal to 1 (for some $n$ ).

- $A$ and $U$ are contractible.

We will now see some examples of groups that are not Lipschitz 1-connected.

\subsection{Groups of SOL Type}

Fix real numbers $t_{2}>1>t_{1}>0$ and consider the group $G$ of matrices of the form

$$
\left[\begin{array}{ccc}
t_{1}^{s} & 0 & x \\
0 & t_{2}^{s} & y \\
0 & 0 & 1
\end{array}\right],
$$

where $s, x, y \in \mathbb{R}$. Note that $G$ decomposes as $U \rtimes A$ as above if we take $A$ to be the diagonal matrices of $G$ and $U$ to be the matrices with diagonal entries equal to 1 . It is known [5, Theorem 8.1.3] that there exist loops $\gamma$ in $G$ such that the minimal area of any filling of $\gamma$ is on the order of $\exp (\operatorname{Lip}(\gamma))$. Hence, $G$ is not Lipschitz 1-connected. Groups of this form are called groups of SOL type.

More generally, Cornulier and Tessera have shown that if a group $G=U \rtimes A$ as above surjects onto a group of SOL type, then it has loops of exponentially large area, and hence cannot be Lipschitz 1-connected [4, Theorem 12.C.1]. If $G$ surjects onto a group of SOL type, we say that $G$ has the SOL obstruction.

\subsection{Tame Groups}

On the other hand, if the conjugation action of some $a \in A$ contracts $U$, then $G=U \rtimes A$ will be Lipschitz 1-connected (Proposition 4.5). For $a$ to be a contraction means that there is some compact subset $\Omega$ of $U$ such that for any compact subset $K$ of $U$, some positive power of $a$ conjugates $K$ into $\Omega$. If such $a$ and $\Omega$ exist, $G$ is said to be tame.

\subsection{The Theorem of Cornulier and Tessera}

It is clear that a space that is Lipschitz 1-connected admits a quadratic isoperimetric inequality, i.e., any loop of length $\ell$ must bound a disk of area $O\left(\ell^{2}\right)$. Cornulier and Tessera have given a large class of solvable Lie groups admitting quadratic isoperimetric inequalities, and we shall extend their result to show that these groups are Lipschitz 1-connected.

Given an action of the abelian group $A$ on a vector space $V$, let $V_{0} \subset V$ be the subspace consisting of vectors $v$ such that $\frac{\log \left\|a^{n} v\right\|}{n} \rightarrow 0$ as $n \rightarrow \infty$ for all $a \in A$. Their theorem [4, Theorem F] states that $G=U \rtimes^{n} A$ satisfies a quadratic isoperimetric inequality if the following conditions hold.

- $(U /[U, U])_{0}=0$. ( $G$ is said to be standard solvable if this condition holds.)

- $G$ does not surject onto a group of SOL type. 
- $H_{2}(\mathfrak{u})_{0}=0$, where $\mathfrak{u}$ is the Lie algebra of $U$ and $H_{2}$ denotes the second Lie algebra homology, see Definition 4.2.

- $\operatorname{Kill}(\mathfrak{u})_{0}=0$, where the Killing module $\operatorname{Kill}(\mathfrak{u})$ is the quotient of the symmetric square $\mathfrak{u} \odot \mathfrak{u}$ of $\mathfrak{u}$ by the $A$-subrepresentation spanned by elements of the form $[x, y] \odot z-y \odot[x, z]$.

Our primary objective in this paper is to establish the following theorem, proved as Theorem 5.1, improving Cornulier and Tessera's result to show Lipschitz 1-connectedness.

Theorem 1.2 Let $G$ be a group of the form $U \rtimes A$, where $U$ and $A$ are contractible real Lie groups, $A$ is abelian, and $U$ is a real unipotent group, i.e., a closed group of strictly upper triangular real matrices.

If $(U /[U, U])_{0}, H_{2}(\mathfrak{u})_{0}$, and $\mathrm{Kill}(\mathfrak{u})_{0}$ are all trivial and $G$ does not surject onto a group of SOL type, then $G$ is Lipschitz 1-connected.

\subsection{Quadratic Isoperimetric Inequality Versus Lipschitz 1-connectedness}

As noted above, if $X$ is Lipschitz 1-connected, then it has a quadratic isoperimetric inequality. It is not known whether the converse is true, that is, there are no known examples where $X$ has a quadratic isoperimetric inequality, but is not Lipschitz 1-connected. Recently, Lytchak, Wenger, and Young [8] showed some results about the existence of Hölder fillings in spaces admitting quadratic isoperimetric inequalities.

\subsection{Organization}

This paper is organized as follows. Section 2 recalls some known results about Lipschitz filling in homogeneous manifolds. Section 3 develops a combinatorial language for describing fillings in Lie groups. Section 4 specializes to solvable Lie groups and reviews the theory of tame groups and Abels's multiamalgam. Finally, we prove our main theorem in Section 5.

\section{Preliminaries}

\subsection{Filling}

In this section, $X$ will be a complete, simply connected Riemannian manifold admitting a transitive Lie group action by isometries. We will collect several facts about filling loops in $X$. Everything in this section is both trivial and well known, but it seems easier to write the proofs than to find them in the literature. The key facts we will prove are as follows. Proposition 2.4 shows that $X$ is Lipschitz 1-connected on a small scale. That is, there is some constant $D$ such that $\operatorname{Span}(\gamma)=O(\operatorname{Lip}(\gamma))$ when $\operatorname{Lip}(\gamma)<D$. Corollary 2.5 shows that all loops in $X$ have Lipschitz fillings. Proposition 2.6 proves the existence a filling span function, meaning that there is a function $C(X, R)$ such that $\operatorname{Span}(\gamma)<C(X, \operatorname{Lip}(\gamma))$ for all Lipschitz loops $\gamma: S^{1} \rightarrow X$. Proposition 2.7 shows that Lipschitz 1-connectedness is a QI-invariant in this setting. 

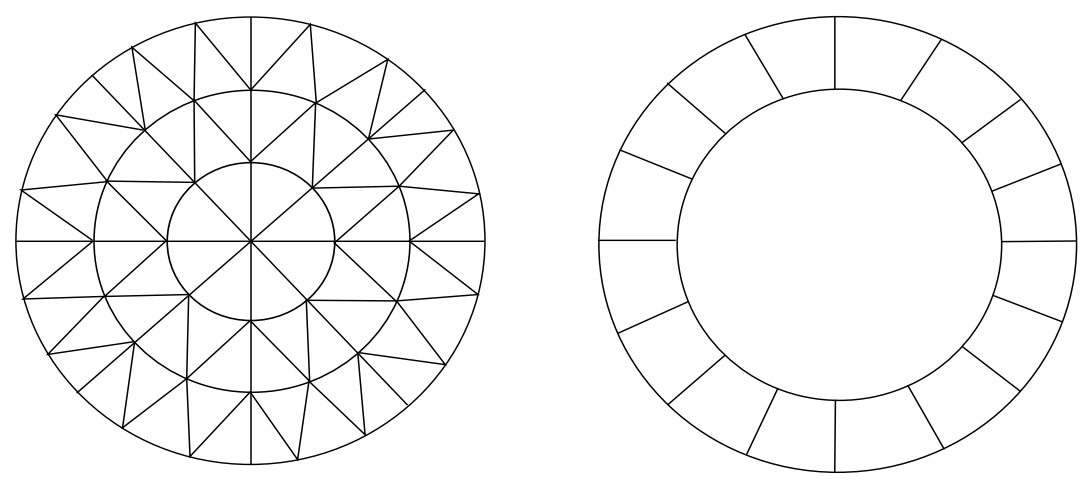

Figure 1: On the right, a decomposition of the unit disk into $O\left(\epsilon^{-2}\right)$ triangles uniformly bilipschitz to $D_{\epsilon}^{2}$ as in Proposition 2.2. On the right, a decomposition of $D^{2}$ into a central disk together with $O\left(\epsilon^{-1}\right)$ sectors uniformly bilipschitz to $D_{\epsilon}^{2}$ as in Proposition 2.3

\subsection{Generalizing to the Cocompact Case}

These results are still true if $X$ is merely assumed to admit a cocompact group action (by isometries) rather than a transitive Lie group action. Although we do not need these generalizations, we will mention them and sketch their proofs for the sake of future researchers.

\subsection{Templates}

Let $D^{2}$ be the unit disk equipped with the Euclidean metric. Purely as a matter of convenience, we will think of $D^{2}$ and the unit circle $S^{1}$ as subsets of $\mathbb{C}$. We will need some convenient cellular decompositions of $D^{2}$.

Definition 2.1 Let $D_{\epsilon}^{2}$ be the Euclidean disk of radius $\epsilon$.

Proposition 2.2 There exists a constant $C$ such that for any $0<\epsilon<1$ there is a triangulation of $D^{2}$ into at most $C \epsilon^{-2}$ triangles that are C-bilipschitz to $D_{\epsilon}^{2}$.

See the left side of Figure 1 for a decomposition of this type. The proof of Proposition 2.2 is left to the reader.

\subsection{Using Templates}

Proposition 2.2 will help us fill loops in the following way. Suppose that $\mathcal{C}$ is a class of loops in $X$ such that $\operatorname{Span}(\gamma)=O(\operatorname{Lip}(\gamma))$ for $\gamma \in \mathcal{C}$; typically, $\mathcal{C}$ will consist of loops having small Lipschitz constant. Now given some other loop $\gamma: S^{1} \rightarrow X$, we may wish to find a $O(\operatorname{Lip}(\gamma))$-Lipschitz filling $f: D^{2} \rightarrow X$ of $\gamma$. We can often use the following abstract strategy to construct $f$. 
- For some sufficiently small $\epsilon$, take a decomposition of $D^{2}$ as in Proposition 2.2. For a 2-cell $\Delta$ of this decomposition, let $\psi_{\Delta}: \Delta \rightarrow D_{\epsilon}^{2}$ be a $C$-bilipschitz map, where $C$ is the universal constant guaranteed by the proposition.

- Take $f$ to be $\gamma$ on $\partial D^{2}$, and extend $f$ over the 1-skeleton of the decomposition in such a way that $\operatorname{Lip}(f)=O(\operatorname{Lip}(\gamma))$ and the restriction of $f$ to the boundary of each 2-cell represents an element of $\mathcal{C}$. That is, we want $f$ to be such that for every 2-cell $\Delta$, the map $\gamma_{\Delta}: S^{1} \rightarrow X$ given by $\gamma_{\Delta}:\left.e^{i \theta} \mapsto f\right|_{\partial \Delta} \circ \psi_{\Delta}^{-1}\left(\epsilon e^{i \theta}\right)$ satisfies $\gamma_{\Delta} \in \mathcal{C}$.

- For each 2-cell $\Delta$, observe that $\operatorname{Lip}\left(\gamma_{\Delta}\right)=O(\epsilon \operatorname{Lip}(\gamma))$, so a $O(\epsilon \operatorname{Lip}(\gamma))$-Lipschitz filling $f_{\Delta}: D^{2} \rightarrow X$ of $\gamma_{\Delta}$ exists.

- Extend $f$ over $\Delta$ with Lipschitz constant $O(\operatorname{Lip}(\gamma))$ by taking, for each 2-cell $\Delta$,

$$
\left.f\right|_{\Delta}(z)=f_{\Delta}\left(\frac{1}{\epsilon} \psi_{\Delta}(z)\right)
$$

The nontrivial part of this type of argument comes when we try to extend $f$ over the 1-skeleton with the desired properties, so we will typically suppress the other details.

Proposition 2.3 There exists a constant $C$ such that, for each $0<\epsilon<1$, the unit disk $D^{2}$ may be cellularly decomposed into an inner disk of radius $1-\epsilon$, surrounded by an annular region divided into 2-cells (we call these sectors) with the following properties.

(i) Each sector is bounded by two radial line segments, an arc of $\partial D^{2}$, and an arc of the boundary of the inner disk.

(ii) Sectors are C-bilipschitz to $D_{\epsilon}^{2}$.

(iii) The number of sectors is between $\frac{1}{C \epsilon}$ and $\frac{C}{\epsilon}$.

See the right side of Figure 1 for a decomposition of this type. The proof of Proposition 2.3 is left to the reader. Typically, this proposition will be used to convert a filling of a loop $\widetilde{\gamma}$ to a filling $f$ of a nearby loop $\gamma$, by taking $f$ restricted to the inner disk to be a slightly rescaled filling of $\widetilde{\gamma}$.

\subsection{Basic Results on Filling in Homogeneous Manifolds}

Proposition 2.4 Let $X$ be a simply connected, complete homogeneous Riemannian manifold. There exist constants $C(X)$ and $D(X)$ such that the following hold.

(i) If $x, y \in X$ and $d(x, y) \leq D(X)$, then there is a unique geodesic in $X$ connecting $x$ to $y$.

(ii) Suppose $\gamma: S^{1} \rightarrow X$ is such that $0<\operatorname{Lip}(\gamma)<D(X)$. Then $\operatorname{Span}(\gamma)<C(X) \operatorname{Lip}(\gamma)$.

Proof It is clear that there is a uniform upper bound on sectional curvatures of $X$, since $X$ is homogeneous. Therefore, $X$ is a CAT( $\kappa)$ space for some $\kappa \geq 0$ [3, Theorem 1A.6]. This implies [3, Proposition 1.4(1)] that there is some constant $D(\kappa)$ depending only on $\kappa$ such that points of $X$ separated by less than $D(\kappa)$ are connected by a unique geodesic, so taking $D(X)<D(\kappa)$ ensures unique geodesics.

By the existence of normal coordinates in Riemannian manifolds [7, Proposition 8.2], there exists a bilipschitz map $\psi: U \rightarrow V$ where $U$ is a neighborhood in $X$ and $V$ a neighborhood in $\mathbb{R}^{\operatorname{dim}(X)}$. Take $D>0$ small enough that there is some $x \in U$ with $U \supset B_{D}(x)$ and $\psi\left(B_{D}(X)\right) \subset V_{0} \subset V$ for some convex set $V_{0}$. Now let $\gamma: S^{1} \rightarrow X$ be 
$D$-Lipschitz with $\gamma(1)=x$. Without loss of generality, $\psi(x)=0$, and we may fill $\psi \circ \gamma$ by coning off, that is, we define a $\operatorname{Lip}(\psi) \operatorname{Lip}(\gamma)$-Lipschitz filling $f_{0}: D^{2} \rightarrow V_{0}$ of $\psi \circ \gamma$ by letting $f_{0}\left(r e^{i \theta}\right)=r \psi\left(\gamma\left(e^{i \theta}\right)\right)$. If we let $f=\psi^{-1} \circ f_{0}$, then $f$ is a filling of $\gamma$ and $\operatorname{Lip}(f) \leq \operatorname{Lip}\left(\psi^{-1}\right) \operatorname{Lip}(\psi) \operatorname{Lip}(\gamma)$. Taking $D(X)<D$ and $C(X)>\operatorname{Lip}\left(\psi^{-1}\right) \operatorname{Lip}(\psi)$, the result follows.

\subsection{Extending Proposition 2.4 to the Cocompact Case}

Proposition 2.4 is still true if, instead of assuming that $X$ admits a transitive Lie group action, we only ask that $X$ admits a cocompact action of some group by isometries. We sketch the proof here. First, it is clear that $X$ still has an upper bound on sectional curvature, and hence sufficiently close points are connected by a unique geodesic by [3, Proposition 1.4] as before. Now, arguing as above, we may cover the compact quotient of $X$ by a finite collection of open neighborhoods $U_{i}$ whose preimages in $X$ are bilipschitz to convex neighborhoods in Euclidean space. Taking $D(X)$ sufficiently small, we see that any $D(X)$ Lipschitz loop $\gamma$ in $X$ must be contained in a lift of a single $U_{i}$, and hence admit a $O(\operatorname{Lip}(\gamma))$ filling by the same argument as above.

Corollary 2.5 For all Lipschitz maps $\gamma: S^{1} \rightarrow X$, we have $\operatorname{Span}(\gamma)<\infty$.

Proof Let $\widetilde{f}: D^{2} \rightarrow X$ be a (continuous) filling of a Lipschitz $\gamma: S^{1} \rightarrow X$. We must find a Lipschitz filling $f$ of $\gamma$. For $\epsilon>0$, let $\tau_{\epsilon}$ be the triangulation of $D^{2}$ given in Proposition 2.2, and for each 2-cell $\Delta$ of $\tau_{\epsilon}$, let $\psi_{\Delta}: \Delta \rightarrow D_{\epsilon}^{2}$ be the $C$-bilipschitz map guaranteed by the proposition. Let $F(\epsilon)$ be the largest value of $d(\widetilde{f}(x), \widetilde{f}(y))$ such that $x, y$ are adjacent vertices of $\tau_{\epsilon}$. Since $f$ is a continuous function on a compact set, $f$ must be uniformly continuous and thus $F(\epsilon) \rightarrow 0$ as $\epsilon \rightarrow 0$.

We will now use Proposition 2.4 to produce a Lipschitz filling $f$ of $\gamma$ as follows. Set $f(x)=\widetilde{f}(x)$ for each vertex $x$ of $\tau_{\epsilon}$, where $\epsilon$ is small enough that $C F(\epsilon)<D(X)$, where $C$ is the constant given by Proposition 2.2 and $D(X)$ is the constant given by Proposition 2.4. Set $f$ to be a constant-speed geodesic on each edge of $\tau_{\epsilon}$, so that the map $S^{1} \rightarrow X$ given by $e^{i \theta} \mapsto f\left(\psi_{\Delta}^{-1}\left(\epsilon e^{i \theta}\right)\right)$ is $D(X)$-Lipschitz. By Proposition 2.4, $f$ now admits a Lipschitz extension over 2-cells.

\subsection{Extending Corollary 2.5 to the Cocompact Case}

If we relax the assumption that $X$ is homogeneous, and instead assume that $X$ admits a compact quotient, Corollary 2.5 is still true, because, as noted above, Proposition 2.4 is still true for such $X$.

Proposition 2.6 Let $X$ be a simply connected, complete homogeneous Riemannian manifold. For any $L>0$, there exists $C(X, L)>0$ such that if $\gamma: S^{1} \rightarrow X$ is L-Lipschitz, then $\operatorname{Span}(\gamma) \leq C(X, L)$.

Gromov [6, $₫ 5]$ referred to $C(X, L)$ as the filling span function of $X$.

Proof Fix $L$ (henceforth, all constants will be presumed to depend uncontrollably on $L$ ). Let $\mathcal{C}_{L}$ denote the $L$-Lipschitz loops in $X$ with some fixed basepoint $x$, and 
equip $\mathcal{C}_{L}$ with the uniform metric. By Arzela-Ascoli, $\mathcal{C}_{L}$ is compact. Certainly, Span is not continuous on $\mathcal{C}_{L}$ because there exists $\gamma \in \mathcal{C}_{L}$ arbitrarily close to the constant map with $L=\operatorname{Lip}(\gamma) \leq \operatorname{Span}(\gamma)$, but we will show that it is bounded. Because $\mathcal{C}_{L}$ is compact, for every $\epsilon>0, \mathcal{C}_{L}$ may be covered by a finite number of $\epsilon$ balls. Hence, it suffices to find a constant $C>1$ such that for $\gamma_{0}, \gamma \in \mathcal{C}_{L}$ with $d\left(\gamma, \gamma_{0}\right)<\frac{1}{C}$ we have

$$
\operatorname{Span}(\gamma)<C \max \left\{1, \operatorname{Span}\left(\gamma_{0}\right)\right\}
$$

To do this, let $D(X)$ be as in Proposition 2.4 (assuming without loss of generality that $D(X)<1)$, and let $\gamma_{0}, \gamma \in \mathcal{C}_{L}$ with $d\left(\gamma_{0}, \gamma\right)<D(X)$. Let $f_{0}: D^{2} \rightarrow X$ be a Lipschitz filling of $\gamma_{0}$. Let $\epsilon=\frac{D(X)}{L}$ and decompose $D^{2}$ into an inner disk and sectors as in Proposition 2.3. We will produce a filling $f: D^{2} \rightarrow X$ of $\gamma$ that is $\frac{\operatorname{Lip}\left(f_{0}\right)}{1-\epsilon}$-Lipschitz on the inner disk and $O(1)$-Lipschitz on the annular region as desired.

Define $\left.f\right|_{S^{1}}$ to be equal to $\gamma$. Let $R=1-\epsilon$ be the radius of the inner disk and define $f$ to be a rescaled copy of $f_{0}$ on the inner disk, i.e., $f\left(r e^{i \theta}\right)=f_{0}\left(\frac{r}{R} e^{i \theta}\right)$ for $r \leq R$. This implies that $f$ is $\frac{\operatorname{Lip}\left(f_{0}\right)}{R}$-Lipschitz on the inner disk. If $x$ and $y$ are the images under $f$ of the endpoints of a radial segment separating two sectors, then $d(x, y)<D(X)$, because $d\left(\gamma, \gamma_{0}\right)<D(X)$. Define $f$ to be a minimal speed geodesic on each of the radial segments separating two sectors, so that $f$ is $O(1)$-Lipschitz on the boundary of each sector (considering $L$ as fixed). Since sectors are uniformly bilipschitz to $D_{\epsilon}^{2}$, $f$ admits a $O(1)$-Lipschitz extension over each sector by Proposition 2.4.

We see that $f$ is $O(1)$-Lipschitz on the annular region and $\frac{\operatorname{Lip}\left(f_{0}\right)}{1-\epsilon}$-Lipschitz on the inner disk, implying the desired bound for $\operatorname{Span}(\gamma)$, taking any sufficiently large $C$.

\subsection{Generalizing Proposition 2.6 to the Cocompact Case}

Because Proposition 2.4 and Corollary 2.5 remain true if $X$ is assumed to be cocompact rather than homogeneous, the proof of Proposition 2.6 remains valid in this case, except that we must take $\mathcal{C}_{L}$ to consist of all $L$-Lipschitz loops in $X$ based in some compact set $K \subset X$ that projects surjectively onto the cocompact quotient of $X$.

Proposition 2.7 Let $X$ and $Y$ be simply connected, complete, homogeneous Riemannian manifolds, and suppose that $X$ is quasi-isometric to $Y$. If $Y$ is Lipschitz 1-connected, then so is $X$.

Proof Let $\psi: X \rightarrow Y$ be a quasi isometry and $\Psi: Y \rightarrow X$ a quasi inverse to $\psi$. Let $\gamma: S^{1} \rightarrow X$ be an $L$-Lipschitz loop, where $L>1$ without loss of generality, and let $\epsilon=\frac{1}{L}$. To obtain a $O(L)$-Lipschitz filling for $\gamma$, we proceed as follows.

Using Proposition 2.2, subdivide $D^{2}$ into $O\left(L^{2}\right)$ triangles bilipschitz to $D_{\epsilon}^{2}$, so that adjacent vertices on the boundary are mapped to within $O(1)$ of each other by $\psi \circ \gamma$. Let $\widetilde{\gamma}: S^{1} \rightarrow Y$ be a $O(L)$-Lipschitz loop in $Y$ that agrees with $\psi \circ \gamma$ on vertices of our triangulation. By assumption, $Y$ is Lipschitz 1-connected, so $\widetilde{\gamma}$ admits a $O(L)$-Lipschitz filling $\widetilde{f}: D^{2} \rightarrow Y$.

We wish to convert $\Psi \circ \tilde{f}$ into a filling $f: D_{2} \rightarrow X$ of $\gamma$. Let $f_{0}: D^{2} \rightarrow X$ be a $O(L)$-Lipschitz map that agrees with $\Psi \circ \widetilde{f}$ on vertices of our triangulation: such a map exists 
because we can fill edges with constant-speed geodesics and then fill triangles in $X$ by Proposition 2.6, as $\Psi \circ \widetilde{f}$ maps adjacent vertices to within $O(1)$ of each other. This gives us a $O(L)$-Lipschitz filling of $\left.f_{0}\right|_{S^{1}}$. Note that the distance from $\gamma$ to $\left.f_{0}\right|_{S^{1}}$ in the uniform metric is $O(1)$ because $\left.f_{0}\right|_{S^{1}}$ agrees with $\Psi \circ \psi \circ \gamma$ on vertices.

Now we take a new subdivision of $D^{2}$, using Proposition 2.3 to subdivide $D^{2}$ into an inner disk surrounded by $O(L)$ sectors that are uniformly bilipschitz to $D_{\epsilon}^{2}$. We build our filling $f$ of $\gamma$ as follows. On the inner disk, let $f$ be given by a rescaled copy $f_{0}$. On radial segments, take $f$ to be a constant speed geodesic, and fill sectors using Proposition 2.6.

\subsection{Generalizing Proposition 2.7 to the Cocompact Case}

The above proof works even if we assume that $X$ and $Y$ have compact quotients instead of being homogeneous. Indeed, it works for any $X$ and $Y$ admitting filling span functions that go to 0 as $L \rightarrow 0$.

\section{Lipschitz Moves}

We now specialize to the case where $X$ is equal to some simply connected Lie group $G$ equipped with a left-invariant Riemannian metric. Our main goal in this section is to reduce questions about filling loops in $G$ to questions about manipulating words in some compact generating set for $G$.

Notation. Any simply connected Lie group $G$ admits a compact generating set $\mathcal{S}$. The set $\mathcal{S}^{*}$ consists of all words $s_{1} s_{2} \cdots s_{\ell}$ where $s_{1}, \ldots, s_{\ell} \in \mathcal{S}$ and $\ell \in \mathbb{N}$, together with the empty word $\varepsilon$. The length of a word $w \in \mathcal{S}^{*}$ will be denoted $\ell(w)$. Given $w=s_{1} \cdots s_{\ell} \in$ $\mathcal{S}^{*}, w^{-1}$ denotes the word $s_{\ell}^{-1} \cdots s_{1}^{-1}$. If $w \in \mathcal{S}^{*}$ represents the identity element $1_{G}$ of $G, w$ is said to be a relation. If $w, w^{\prime} \in \mathcal{S}^{*}$ represent the same element of $G$, we write $w={ }_{G} w^{\prime}$. The word norm with respect to $\mathcal{S}$ will be denoted by $|\cdot| \delta$. That is, for $g \in G$, we define $|g|_{\mathcal{S}}$ to be $\inf \left\{\ell(w): g={ }_{G} w \in \mathcal{S}^{*}\right\}$.

Assumption Any time we take a compact generating set $\mathcal{S}$ for $G$, we assume without loss of generality that $\mathcal{S}$ is symmetric (meaning that $s \in \mathcal{S}$ if and only if $s^{-1} \in \mathcal{S}$ ) and that $1_{G} \in \mathcal{S}$, unless otherwise indicated.

\subsection{It Suffices to Fill Over the Unit Square}

It will be convenient to consider loops as maps $[0,1] \rightarrow X$, rather than $S^{1} \rightarrow X$, and fillings as maps from the unit square $[0,1] \times[0,1] \rightarrow X$, rather than $D^{2} \rightarrow X$.

Definition 3.1 Given $\beta:[0,1] \rightarrow X$ with $\beta(0)=\beta(1)$, a filling of $\beta$ over the unit square is a map $f:[0,1] \times[0,1] \rightarrow X$ with the following properties:

(i) $f$ agrees with $\beta$ on the bottom edge of the unit square, meaning that $f(t, 0)=$ $\beta(t)$ for all $t \in[0,1]$;

(ii) $f$ is constant on the set on the other three edges of the unit square, meaning that $f(x, y)=\beta(0)$ for all $(x, y) \in([0,1] \times 1) \cup(0 \times[0,1]) \cup(1 \times[0,1])$. 
We define $\operatorname{Span}(\beta)$ to be the infimum of $\operatorname{Lip}(f)$ as $f$ ranges over Lipschitz fillings of $\beta$ over the unit square.

Similarly, given $\beta, \gamma:[0,1] \rightarrow X$, a homotopy from $\beta$ to $\gamma$ is a map $[0,1] \times[0,1] \rightarrow X$ such that $f(t, 1)=\gamma(t), f(t, 0)=\beta(t)$, and the restrictions $\left.f\right|_{0 \times[0,1]}$ and $\left.f\right|_{1 \times[0,1]}$ are constant.

We will now see that it makes no difference whether we consider loops as maps from $[0,1]$ or $S^{1}$. The key tool is the following lemma.

Lemma 3.2 There exists a constant $C$ such that, given loops $\beta: S^{1} \rightarrow X$ and $\gamma: S^{1} \rightarrow X$ such that $\gamma$ is a reparameterization of $\beta$, and given a filling $f: D^{2} \rightarrow X$ of $\gamma$, we have

$$
\operatorname{Span}(\beta) \leq C \max \{\operatorname{Lip}(f), \operatorname{Lip}(\beta)\}
$$

Proof An equivalent statement was proved in [10, Lemma 8.13].

Corollary 3.3 There is a universal constant $C>0$ with the following property. Suppose that $\gamma: S^{1} \rightarrow X$ is a Lipschitz loop and $\beta:[0,1] \rightarrow X$ a Lipschitz path with $\beta(t)=$ $\gamma\left(e^{2 \pi i t}\right)$, for all $t \in[0,1]$. Then $\frac{\operatorname{Span}(\beta)}{C}<\operatorname{Span}(\gamma)<C \operatorname{Span}(\beta)$.

Proof Let $\beta_{0}: \partial\left([0,1]^{2}\right) \rightarrow X$ be equal to $\beta$ on the bottom edge and $\beta(0)$ on the other three edges, and fix a bilipschitz map $\psi: D^{2} \rightarrow[0,1] \times[0,1]$. Observe that $\gamma$ is a reparameterization of $\beta_{0} \circ \psi: S^{1} \rightarrow X$. If $f: D^{2} \rightarrow X$ is a Lipschitz filling of $\gamma$, then by Lemma 3.2 there is some $O(\operatorname{Lip}(f))$-Lipschitz filling $\widetilde{f}: D^{2} \rightarrow X$ of $\beta_{0} \circ \psi$, so $\widetilde{f} \circ \psi^{-1}$ is an $O(\operatorname{Lip}(f))$-Lipschitz filling of $\beta$. We see that $\operatorname{Span}(\beta)=O(\operatorname{Span}(\gamma))$. The reverse inequality is proved similarly.

\subsection{Filling Relations}

\subsubsection{It Suffices to Fill Words}

Let $\mathcal{S}$ be a compact generating set for $G$. For each $s \in \mathcal{S}$, choose a Lipschitz curve $\gamma_{s}:[0,1] \rightarrow G$ connecting 1 to $s$ such that the following properties hold. There is some uniform bound on $\operatorname{Lip}\left(\gamma_{s}\right)$ as $s$ ranges over $\mathcal{S}, \gamma_{1_{G}}$ is constant, $\gamma_{s}(1-t)=\gamma_{s^{-1}}(t)$, for all $s \in \mathcal{S}$ and $t \in[0,1]$. Given a word $w=s_{1} s_{2} \cdots s_{\ell} \in \mathcal{S}^{*}$, let $\gamma_{w}:[0,1] \rightarrow G$ denote the concatenation of the paths $\gamma_{s_{1}}, s_{1} \gamma_{s_{2}}, \ldots, s_{1} \cdots s_{\ell-1} \gamma_{s_{\ell}}$, reparameterized so that the $i$-th of these paths is used on $\left[\frac{i-1}{\ell}, \frac{i}{\ell}\right]$. That is, for $0 \leq t \leq 1$ and $i=1, \ldots \ell$, we have

$$
\gamma_{w}\left(\frac{i-1+t}{\ell}\right)=s_{1} s_{2} \cdots s_{i-1} \gamma_{s_{i}}(t)
$$

Proposition 3.4 Suppose that there exists a constant $C$ such that for every $w \in \mathcal{S}^{*}$ with $w={ }_{G} 1_{G}$, there exists a $C \ell(w)$-Lipschitz filling of $\gamma_{w}$ over the unit square. Then $G$ is Lipschitz 1-connected.

Proof Note that $G=\bigcup_{k=1}^{\infty} \mathcal{S}^{k}$, and each $\mathcal{S}^{k}$ is compact. Hence, by the Baire category theorem, some power $\mathcal{S}^{k}$ must contain an open neighborhood, so $\mathcal{S}^{2 k}$ contains some 
open neighborhood of the identity, which in turn contains the $r$-ball around the identity for some $r>0$. Take $K$ to be a natural number larger than $\frac{2 k}{r}$, so that $\mathcal{S}^{K}$ contains the 1-ball around the identity in $G$.

Given a loop $\gamma:[0,1] \rightarrow G$, we produce an $O(\operatorname{Lip}(\gamma))$-Lipschitz filling $f:[0,1]^{2} \rightarrow G$ of $\gamma$ as follows. Let $n=\lceil\operatorname{Lip}(\gamma)\rceil$. By Propositions 2.4 and 2.6 it suffices to consider only $\gamma$ such that $n$ is at least 2 . Cellularly decompose the unit square into the rectangle $[0,1] \times\left[\frac{1}{n}, 1\right]$ together with the collection of squares $\left[\frac{i}{n}, \frac{i+1}{n}\right] \times\left[0, \frac{1}{n}\right]$ as $i$ runs from 0 to $n-1$.

Define $f$ to be constant on all the vertical edges. Note that for each $i$, the element $\gamma\left(\frac{i}{n}\right)^{-1} \gamma\left(\frac{i+1}{n}\right)$ lies in the 1-ball in $G$, so it may be represented by some $w_{i} \in \mathcal{S}^{K}$. On the horizontal edge connecting $\left(\frac{i}{n}, \frac{1}{n}\right)$ to $\left(\frac{i+1}{n}, \frac{1}{n}\right)$, let $f$ be a copy of $\gamma_{w_{i}}$ translated by $\gamma\left(\frac{i}{n}\right)$. that is, for $0 \leq t \leq 1$, take $f\left(\frac{i+t}{n}, \frac{1}{n}\right)=\gamma\left(\frac{i}{n}\right) \gamma_{w_{i}}(t)$. Letting $w=$ $w_{1} \cdots w_{n}$, observe that $f$ agrees with $\gamma_{w}$, the bottom of the rectangle $[0,1] \times\left[\frac{1}{n}, 1\right]$, so $f$ may be extended over this rectangle with $\operatorname{Lip}(f)=O\left(\operatorname{Lip}\left(\gamma_{w}\right)\right)=O(\ell(w))=$ $O(\operatorname{Lip}(\gamma))$, where the first bound comes from our hypothesis. Finally, $f$ admits a $O(\operatorname{Lip}(\gamma))$-Lipschitz extension over each square by Proposition 2.6.

Definition 3.5 Suppose $\mathcal{C}$ is a collection of pairs $(v, w)$, where $v, w \in \mathcal{S}^{*}$ are words in $\mathcal{S}$. We say that $v \leadsto w$ for $(v, w) \in \mathcal{C}$ if there exists some constant $C$ depending only on $\mathcal{C}$ with the following property: given $(v, w) \in \mathcal{C}$, the map from $\partial([0,1] \times[0,1])$ to $X$ that is equal to $\gamma_{v}$ on the bottom edge, $\gamma_{w}$ on the top edge, and constant on the sides admits a $C \ell(v)$-Lipschitz filling.

We now give some basic rules for manipulating fillings. Often, the set $\mathcal{C}$ will be inferred from context.

Lemma 3.6 The following rules hold in any simply connected Lie group G.

(i) Suppose $\mathcal{C} \subset \mathcal{S}^{*} \times \mathcal{S}^{*}$. Then $v \leadsto w$ for $(v, w) \in \mathcal{C}$ if and only if $v w \leadsto \varepsilon$ for $(v, w) \in \mathcal{C}$ and $\operatorname{Lip}(w)=O(\operatorname{Lip}(v))$, for each pair $(v, w) \in \mathcal{C}$.

(ii) Fix $n$, and suppose given sets $\mathcal{C}_{i} \subset \mathcal{S}^{*} \times \mathcal{S}^{*}$ for $i=1$ to $n$. If, for each $i=1$ to $n$, we have $v \leadsto w$, for $(v, w) \in \mathcal{C}_{i}$, then $v_{1} \cdots v_{n} \leadsto w_{1} \cdots w_{n}$, for all $\left(v_{1}, w_{1}\right) \in$ $\mathcal{C}_{1}, \ldots,\left(v_{n}, w_{n}\right) \in \mathcal{C}_{n}$.

(iii) Given $\mathcal{C}, \mathrm{C}^{\prime} \subset \mathcal{S}^{*} \times \mathcal{S}^{*}$, if $v_{1} \leadsto v_{2}$ for $\left(v_{1}, v_{2}\right) \in \mathcal{C}$ and $v_{2} \leadsto v_{3}$ for $\left(v_{2}, v_{3}\right) \in \mathcal{C}^{\prime}$, then $v_{1} \leadsto v_{3}$ for pairs $\left(v_{1}, v_{3}\right)$ such that there exists $v_{2} \in \mathcal{S}^{*}$ with $\left(v_{1}, v_{2}\right) \in \mathcal{C}$ and $\left(v_{2}, v_{3}\right) \in \mathcal{C}^{\prime}$.

(iv) $w w^{-1} \leadsto \varepsilon$ for $w \in \mathcal{S}^{*}$.

(v) Let $U \subset G$ be a bounded neighborhood of the identity in $G$. If $\mathcal{D}$ is the collection of relations $w=s_{1} \cdots s_{\ell} \in \mathcal{S}^{*}$ such every prefix $s_{1} \cdots s_{i}$ represents an element of $U$, then $w \leadsto \varepsilon$ for $w \in \mathcal{D}$.

(vi) $G$ is Lipschitz 1-connected if and only if $v \leadsto \varepsilon$ for every relation $v \in \mathcal{S}^{*}$.

Proof (i) follows immediately from Lemma 3.2.

To prove (ii), subdivide the unit square into rectangles of the form $\left[\frac{i-1}{n}, \frac{i}{n}\right] \times[0,1]$ for $i=1, \ldots, n$. Suppose $\left(v_{1}, w_{1}\right) \in \mathcal{C}_{1}, \ldots,\left(v_{n}, w_{n}\right) \in \mathcal{C}_{n}$ are given. By hypothesis, there exists a homotopy $f_{i}:[0,1] \times[0,1] \rightarrow G$ from $v_{i}$ to $w_{i}$ with $\operatorname{Lip}\left(f_{i}\right)=O\left(\operatorname{Lip}\left(v_{i}\right)\right)$. 
Define a map $f:[0,1] \times[0,1]$ by putting a rescaled copy of $f_{i}$ in $\left[\frac{i-1}{n}, \frac{i}{n}\right] \times[0,1]$, that is, for $0 \leq t \leq 1$ and $i=1, \ldots, n$, we take $f\left(\frac{i-1+t}{n}, y\right)=f_{i}(t, y)$. Thus $\operatorname{Lip}(f)=$ $O\left(\max \left\{n \operatorname{Lip}\left(f_{i}\right): i=1, \ldots, n\right\}\right)=O(\operatorname{Lip}(v))$ because $n$ is fixed. Since $f$ restricted to the top of the unit square is a reparameterization of $v_{1} \cdots v_{n}$, and $f$ restricted to the bottom of the unit square is a reparameterization of $w_{1} \cdots w_{n}$, we are done.

The proof of (iii) is similar: divide the unit square into two rectangles $[0,1] \times\left[0, \frac{1}{2}\right]$ and $[0,1] \times\left[\frac{1}{2}, 1\right]$ and put a rescaled copy of the homotopy from $v_{1}$ to $v_{2}$ into the bottom rectangle and a rescaled copy of the homotopy from $v_{2}$ to $v_{3}$ into the top rectangle to get the desired homotopy $v_{1}$ to $v_{3}$.

To prove (iv), let $w \in \mathcal{S}^{*}$ and let $f:[0,1] \times[0,1]$ be defined by

$$
f(t, s)=\gamma_{w}(2 \max \{0, \min \{t-s, 1-t-s\}\})
$$

The reader may check that $f$ is a $\operatorname{Lip}\left(\gamma_{w w^{-1}}\right)$-Lipschitz filling of $\gamma_{w w^{-1}}$.

To prove (v), let $K$ be such that $\mathcal{S}^{K}$ contains $U$. Given $w \in \mathcal{D}$, let $\ell=\ell(w)$, so that we can write $w=s_{1} \cdots s_{\ell}$. We will find an $O(\ell)$ filling $f$ of $\gamma_{w}$ as follows. Subdivide $[0,1] \times[0,1]$ into the rectangle $[0,1] \times\left[\frac{1}{\ell}, 1\right]$ together with the squares of the form $\left[\frac{i-1}{\ell}, \frac{i}{\ell}\right] \times\left[0, \frac{1}{\ell}\right]$. Define $f$ to be constant on $[0,1] \times\left[\frac{1}{\ell}, 1\right]$. For each $i$, choose $w_{i} \in \mathcal{S}^{K}$ such that $w_{i}={ }_{G} s_{1} \cdots s_{i}$ and take $f$ to be $\gamma_{w_{i}}$ on the vertical edge $\frac{i}{\ell} \times\left[0, \frac{1}{\ell}\right]$, that is $f\left(\frac{i}{\ell}, \frac{1-t}{\ell}\right)=\gamma_{w_{i}}(t)$, for $0 \leq t \leq 1$ and $i=0, \ldots, n$. We may extend $f$ over each square with Lipschitz constant $O(\ell)$ by Proposition 2.6.

(vi) is a consequence of Proposition 3.4.

\subsection{Normal Form Triangles}

We now discuss normal forms $\omega$ and $\omega$-triangles. Using a technique of Gromov, we shall see that $G$ is Lipschitz 1-connected if $\omega$-triangles are Lipschitz 1-connected.

Definition 3.7 A normal form for a compactly generated group $G$ equipped with compact generating set $\mathcal{S}$ is a map $\omega: G \rightarrow \mathcal{S}^{*}$ such that $\ell(\omega(g))=O\left(|g|_{\mathcal{S}}\right)$ for $g \in G$. If $\omega$ is a normal form, then an $\omega$-triangle is a word in $\mathcal{S}^{*}$ of the form $\omega\left(g_{1}\right) \omega\left(g_{2}\right) \omega\left(g_{3}\right)$, where $g_{1} g_{2} g_{3}={ }_{G} 1$.

Lemma 3.8 Let $\omega: G \rightarrow \mathcal{S}^{*}$ be a normal form. If $\Delta \sim \varepsilon$ for $\omega$-triangles $\Delta$, then $G$ is Lipschitz 1-connected.

Proof This was proved in [10, Proposition 8.14]. The proof is sketched in Figure 2.

\section{Tame Subgroups and the Multiamalgam}

Assumption From here on, we specialize to the case where $G=U \rtimes A$, where $U$ and $A$ are contractible Lie groups, with $A$ abelian and $U$ a closed group of strictly upper triangular real matrices. 


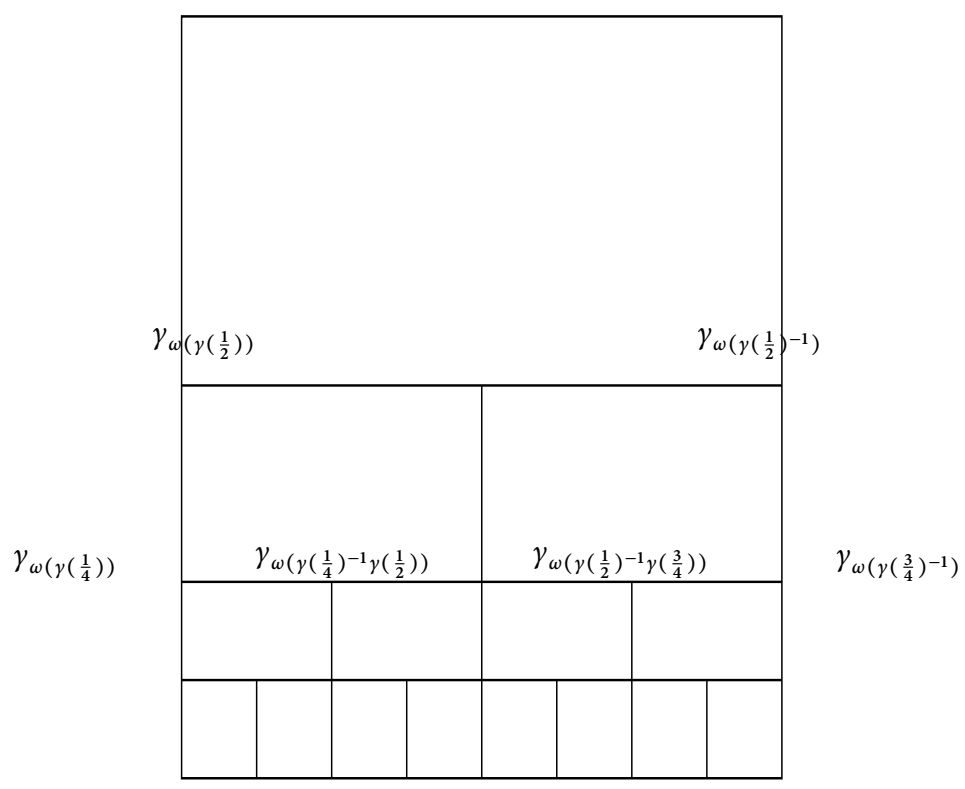

Figure 2: This figure indicates how to fill $\gamma=\gamma_{w}$ for an arbitrary relation $w \in \mathcal{S}^{*}$, given that one knows how to fill $\omega$-triangles. The top edge and all the vertical edges are taken to be constant, and each horizontal edge is understood to be an appropriate translate of its label, so that each rectangle represents an $\omega$-triangle, except the bottom row. The bottom edge is taken to be $\gamma_{w}$, and there is a row of squares along the bottom that can be filled by Proposition 2.6

\subsection{Standard Solvable Groups}

Observe that $A$ acts on the abelianization $U /[U, U]$. Fix a norm on the vector space $U /[U, U]$. If there is no vector $X$ such that $\lim _{n \rightarrow \infty} \frac{1}{n} \log \left\|a^{n} \cdot X\right\| \rightarrow 0$, for all $a \in A$, we say that $G$ is standard solvable.

In this section we will be interested in the structure and geometry of standard solvable groups. Section 4.2 will describe the so-called standard tame subgroups of a standard solvable group. Lemma 4.9 will show how to find Lipschitz fillings for words that already represent the identity in the free product of the standard tame subgroups. Theorem 4.12, quoted from [4], will give conditions under which $G$ can be presented as the free product of its standard tame subgroups modulo certain easily understood amalgamation relations.

\subsection{Weights}

Let $G=U \rtimes A$ be standard solvable, and let $\mathfrak{u}$ be the Lie algebra of $U$, identified as usual with the tangent space of $U$ at $1_{U}$, and fix any norm $\|\cdot\|$ on $\mathfrak{u}$. For $a \in A$, we denote 
the conjugation action of $a$ on $\mathfrak{u}$ by $\operatorname{Ad}(a)$, so that $\operatorname{Ad}(a) X=\left.\frac{d}{d t}\right|_{t=0} a^{-1} \exp (t X) a$. Observe that $\operatorname{Hom}(A, \mathbb{R})$ is a vector space.

Definition 4.1 $([4, \$ 4 . \mathrm{B}]) \quad$ For a homomorphism $\alpha: A \rightarrow \mathbb{R}$, define the $\alpha$-weight space $\mathfrak{u}_{\alpha} \subset \mathfrak{u}$ to consist of 0 together with all $X \in \mathfrak{u}$ such that for all $a \in A$,

$$
\lim _{n \rightarrow \infty} \frac{1}{n} \log \left\|\operatorname{Ad}(a)^{n} X\right\|=\alpha(a) .
$$

Define the set of weights $\mathcal{W}$ to consist of all $\alpha \in \operatorname{Hom}(A, \mathbb{R})$ for which $\operatorname{dim} \mathfrak{u}_{\alpha}>0$. By a conic subset, we mean the intersection of $\mathcal{W}$ with an open, convex cone in $\operatorname{Hom}(A, \mathbb{R})$ that does not contain 0 . Denote the set of all conic subsets by $\mathcal{C}$. For $C \in \mathcal{C}$, let $U_{C}$ denote the closed connected subgroup of $U$ whose Lie algebra is $\oplus_{\alpha \in C} \mathfrak{u}_{\alpha}$, and let $G_{C}=U_{C} \rtimes A$. These groups $G_{C}$ are referred to as standard tame subgroups of $G$ (see remarks in $\$ 4.4)$.

As an exercise, the reader may wish to compute the weights and weight spaces for a group of SOL type. We have that $\mathcal{C}$ is finite, that $\mathfrak{u}=\bigoplus_{\alpha \in \mathcal{W}} \mathfrak{u}_{\alpha}$, and that $\left[\mathfrak{u}_{\alpha}, \mathfrak{u}_{\beta}\right] \subset$ $\mathfrak{u}_{\alpha+\beta}$ for $\alpha, \beta \in \mathcal{W}\left[4, \S 4\right.$.B]. We now define $H_{2}(\mathfrak{u})$ and recall the definition of $\operatorname{Kill}(\mathfrak{u})$ so that we will be able to state Theorem 5.1, our main theorem.

Definition 4.2 Let $d_{3}: \bigwedge^{3} \mathfrak{u} \rightarrow \wedge^{2} \mathfrak{u}$ and $d_{2}: \bigwedge^{2} \mathfrak{u} \rightarrow \mathfrak{u}$ be the maps of $A$-modules induced by taking

$$
d_{3}(x \wedge y \wedge z)=[x, y] \wedge z+[y, z] \wedge x+[z, x] \wedge y \quad d_{2}(x \wedge y)=-[x, y] .
$$

Define $H_{2}(\mathfrak{u})=\operatorname{ker}\left(d_{2}\right) / \operatorname{image}\left(d_{3}\right)$. Define Kill $(\mathfrak{u})$ to be the quotient of the symmetric square $\mathfrak{u} \odot \mathfrak{u}$ by the subspace spanned by elements of the form $[x, y] \odot z-y \odot[x, z]$.

\section{3 $H_{2}(\mathfrak{u})$ and $\operatorname{Kill}(\mathfrak{u})$ Are $A$-representations}

Observe that the natural $A$-action on $\bigwedge^{3} \mathfrak{u}$ descends to an $A$-action on $H_{2}(\mathfrak{u})$ because, by the Jacobi-like identity

$$
\operatorname{Ad}(a)[X, Y]=[\operatorname{Ad}(a) X, Y]+[X, \operatorname{Ad}(a) Y]
$$

the subspaces image $\left(d_{3}\right)$ and $\operatorname{ker}\left(d_{2}\right)$ are preserved by the action of $A$. Similarly, $\mathrm{Kill}(\mathfrak{u})$ is also an $A$-representation. Recall that for an $A$-representation $V$ we define $V_{0}$ to consist of 0 together with vectors $X$ such that $\lim _{n \rightarrow \infty} \frac{1}{n} \log \left\|a^{n} \cdot X\right\|=0$ for all $a \in A$. We thus define the subspaces $H_{2}(\mathfrak{u})_{0}$ and $\operatorname{Kill}(\mathfrak{u})_{0}$.

\subsection{Tame Subgroups}

Definition 4.3 Given $a \in A$, a vacuum subset for $a$ is a compact $\Omega \subset U$ such that for every compact $K \subset U$, there is some $n>0$ with $\operatorname{Ad}(a)^{n} K \subset \Omega$. We say that $G$ is tame if there exists $a \in A$ with a vacuum subset.

We say that $G$ is tame if and only if there is some $a \in A$ with $\alpha(a)<0$ for all $\alpha \in \mathcal{W}$, so $G_{C}$ is tame for $C \in \mathcal{C}$ [4, Proposition 4.B.5]. We now wish to show that if $G$ is tame, then it is Lipschitz 1-connected. Our starting point is the following. 
Proposition 4.4 Suppose $G$ is tame. Then there is some $a \in A$ and a compact generating set $\mathcal{S} \subset U$ for $U$ such that $\operatorname{Ad}(a) \mathcal{S}^{2} \subset \mathcal{S}$.

Proof By hypothesis, there is some $b \in A$ with a vacuum subset $\Omega$. Let $\mathcal{S}_{0}$ be a compact generating set for $U$. As in the proof of Proposition 3.4, we see that some power of $\mathcal{S}_{0}$ contains an open ball around the identity. Hence, for some $M>0$, the set $\mathcal{S}_{0}^{M}$ contains $\Omega$. As $\Omega$ is a vacuum set for $b$, there exists $L$ such that $\operatorname{Ad}(b)^{L} \mathcal{S}_{0}^{2 M} \subset \Omega$. Taking $a=b^{L}$ and $\mathcal{S}=\mathcal{S}_{0}^{M}$, we have that $\mathcal{S}$ is a generating set because it contains $\mathcal{S}_{0}$ (because $1 \in \mathcal{S}_{0}$ by our standing assumption that generating sets contain the identity), and $\operatorname{Ad}(a) \mathcal{S}^{2}=\operatorname{Ad}(b)^{L} \mathcal{S}_{0}^{2 M} \subset \Omega \subset \mathcal{S}$ as desired.

Proposition 4.5 If $G=U \rtimes A$ is tame, then $G$ is Lipschitz 1-connected.

Remark A tame group $G$ is probably CAT( 0 ) for some choice of metric, but we do not know how to prove this, so we give a combinatorial proof using Lemma 3.8.

Proof As in Proposition 4.4, fix $a \in A$ and a compact generating set $\mathcal{S}_{U} \subset U$ such that $\operatorname{Ad}(a) \mathcal{S}_{U}^{2} \subset \mathcal{S}_{U}$. Let $\mathcal{S}_{A}$ be a generating set for $A$ with $a \in \mathcal{S}_{A}$, and let $\mathcal{S}=\mathcal{S}_{U} \cup \mathcal{S}_{A}$, so that $\mathcal{S}$ is a generating set for $G$. Note that $\operatorname{Ad}(a)(s)=s$ for $s \in \mathcal{S}_{A}$, and observe that if $\ell>\left\lceil\log _{2} j\right\rceil>0$, then

$$
\begin{aligned}
\operatorname{Ad}(a)^{\ell}\left(\mathcal{S}_{U}^{j}\right) \subset \operatorname{Ad}(a)^{\ell-1}\left(\mathcal{S}_{U}^{\left[\frac{j}{2}\right]}\right) \subset \operatorname{Ad}(a)^{\ell-2}\left(\mathcal{S}_{U}^{\left[\frac{1}{2}\left[\frac{j}{2}\right]\right\rceil}\right) & \subset \cdots \\
\cdots & \subset \operatorname{Ad}(a)^{\ell-\left[\log _{2} j\right]}\left(\mathcal{S}_{U}\right) \subset \mathcal{S}_{U},
\end{aligned}
$$

because the function $f: j \mapsto\left\lceil\frac{j}{2}\right\rceil$ satisfies $f^{\left[\log _{2} j\right\rceil} j=1$ for natural numbers $j$. In other words, given $u \in U$, there exist $k \leq\left\lceil\log _{2}|u|_{\mathcal{S}_{U}}\right\rceil$ and $s \in \mathcal{S}_{U}$ such that $u={ }_{G} a^{k} s a^{-k}$. It follows, letting $\phi_{A}: G \rightarrow A$ denote projection, that we may define a normal form $\omega: G \rightarrow \mathcal{S}^{*}$ such that

- for any $g \in G$, the word $\omega(g)$ is given by $\omega\left(\phi_{A}(g)\right) \omega\left(\phi_{A}(g)^{-1} g\right)$,

- for $g \in A$, the word $\omega(g) \in \mathcal{S}_{A}^{*}$ is a minimal length word representing $g$,

- and for $g \in U \backslash\{1\}, \omega(g)$ is of the form $a^{k} s a^{-k}$, where $s \in \mathcal{S}_{U}$ and $0 \leq k=$ $O\left(\log |g|_{S_{U}}\right)$.

To check that this is a normal form, i.e., that $\ell(\omega(g))=O\left(|g|_{\delta}\right)$, note that $\left|\phi_{A}(g)\right|_{\delta} \leq$ $|g|_{\mathcal{S}}$ and $\left|\phi_{A}(g)^{-1} g\right|_{S_{U}}=O\left(\exp |g|_{\mathcal{S}}\right)$, so that

$$
\left|\phi_{A}(g)^{-1}(g)\right|_{\mathcal{S}}=O\left(\log \left(O\left(\exp |g|_{\mathcal{S}}\right)\right)\right)=O\left(|g|_{\mathcal{S}}\right),
$$

because $U$ is at most exponentially distorted in $G$ [4, Proposition 6.B.2].

It will suffice to show that $\Delta \leadsto \varepsilon$ for $\omega$-triangles $\Delta \in \mathcal{S}^{*}$. An $\omega$-triangle $\Delta$ has the form $a_{1} \omega\left(u_{1}\right) a_{2} \omega\left(u_{2}\right) a_{3} \omega\left(u_{3}\right)$, where for $i=1,2,3$, we have $u_{i} \in U$ and $a_{i} \in \mathcal{S}_{A}^{*}$ with the $a_{i}$ and $u_{i}$ satisfying $\left(\operatorname{Ad}\left(a_{3} a_{2}\right) u_{1}\right)\left(\operatorname{Ad}\left(a_{3}\right) u_{2}\right) u_{3}=1$. To show that $\Delta \sim \varepsilon$, it thus suffices to establish the following two facts:

- $\omega(u) b \leadsto b \omega\left(b^{-1} u b\right)$ for $u \in U$ and $b \in \mathcal{S}_{A}^{*}$,

- $\omega\left(u_{1}\right) \omega\left(u_{2}\right) \omega\left(u_{3}\right) \leadsto \varepsilon$ for $u_{1}, u_{2}, u_{3} \in U$ such that $u_{1} u_{2} u_{3}=1$. 
Lemma 3.6 will be crucial for showing the first fact, in particular, we use the lemma to provide homotopies between words that stay inside a bounded neighborhood of the identity.

\subsection{Conjugation}

To show that $\omega(u) b \leadsto b \omega\left(b^{-1} u b\right)$ for $u \in U$ and $b \in \mathcal{S}_{A}^{*}$, note that $\omega(u)$ may be written as $a^{k} s a^{-k}$ for some $s \in \mathcal{S}_{U}$ and $k \geq 0$. Because $\operatorname{Ad}(a) \mathcal{S}^{2} \subset \mathcal{S}$, there is some $C \geq 1$ such that, for all $a^{\prime} \in \mathcal{S}_{A}$, we have $\operatorname{Ad}(a)^{C} \operatorname{Ad}\left(a^{\prime}\right) \mathcal{S} \subset \mathcal{S}$. Let $K=C \ell(b)+k$, so that $\operatorname{Ad}(a)^{K-k} \operatorname{Ad}\left(b^{\prime}\right) \mathcal{S} \subset \mathcal{S}$ for any word $b^{\prime} \in \mathcal{S}_{A}^{*}$ with $\ell\left(b^{\prime}\right) \leq \ell(b)$, and let $s^{\prime}, s^{\prime \prime} \in \mathcal{S}$ be given by $s^{\prime}=\operatorname{Ad}(a)^{K-k} s$ and $s^{\prime \prime}=b^{-1} s^{\prime} b$. We homotope as follows, liberally using Lemma 3.6.

$$
\begin{aligned}
\omega(u) b=a^{k} s a^{-k} b & \leadsto a^{K} a^{k-K} s a^{K-k} a^{-K} b \\
& \leadsto a^{K} s^{\prime} b a^{-K} \leadsto a^{K} b b^{-1} s^{\prime} b a^{-K} \leadsto b a^{K} s^{\prime \prime} a^{-K} \leadsto b \omega\left(b^{-1} u b\right) .
\end{aligned}
$$

\subsection{Filling $\omega$-triangles in $U$}

To show that $\omega\left(u_{1}\right) \omega\left(u_{2}\right) \omega\left(u_{3}\right) \leadsto \varepsilon$ for $u_{1}, u_{2}, u_{3} \in U$ such that $u_{1} u_{2} u_{3}=1$, write $\omega\left(u_{i}\right)$ as $a^{k_{i}} s_{i} a^{-k_{i}}$ for $i=1,2,3$, with $k_{i} \geq 0$ and $s_{i} \in \mathcal{S}_{U}$. Let $K=k_{1}+k_{2}+k_{3}$ and let $s_{i}^{\prime}=\operatorname{Ad}\left(a^{k_{i}-K}\right) s_{i} \in \mathcal{S}_{U}$. We homotope as follows.

$$
\begin{aligned}
& \omega\left(u_{1}\right) \omega\left(u_{2}\right) \omega\left(u_{3}\right)=\left(a^{k_{1}} s_{1} a^{-k_{1}}\right)\left(a^{k_{2}} s_{2} a^{-k_{2}}\right)\left(a^{k_{3}} s_{3} a^{-k_{3}}\right) \\
& \sim\left(a^{K} a^{k_{1}-K} s_{1} a^{K-k_{1}} a^{-K}\right)\left(a^{K} a^{k_{2}-K} s_{2} a^{K-k_{2}} a^{-K}\right)\left(a^{K} a^{k_{3}-K} s_{3} a^{K-k_{3}} a^{-K}\right) \\
& \quad \sim\left(a^{K} s_{1}^{\prime} a^{-K}\right)\left(a^{K} s_{2}^{\prime} a^{-K}\right)\left(a^{K} s_{3}^{\prime} a^{-K}\right) \leadsto a^{K} s_{1}^{\prime} s_{2}^{\prime} s_{3}^{\prime} a^{-K} \leadsto a^{K} a^{-K} \leadsto \varepsilon .
\end{aligned}
$$

\subsection{Filling Freely Trivial Words}

We now return to the case where the standard solvable group $G=U \rtimes A$ is not necessarily tame. Recall that the collection of conic subsets $\mathcal{C}$ is finite. For $C \in \mathcal{C}$, let $G_{C}$ be the tame group $U_{C} \rtimes A$, and let $\mathcal{S}_{G_{C}}$ be a compact generating set for this group. Let $H=*_{C \in \mathcal{C}} G_{C}$, and let $\mathcal{S}_{H} \subset H$ be the union of the $\mathcal{S}_{G_{C}}$. There is a natural map from $H$ to $G$. Lemma 4.9 will show that if $w \in \mathcal{S}_{H}^{*}$ represents the identity in $H$, then its image in $G$ admits an $O(\ell(w))$-Lipschitz filling.

We will need the following auxiliary results first. (The reader should probably skip directly to the proof of Lemma 4.9 to understand the point of these propositions). Proposition 4.6 shows that a word $w \in \mathcal{S}_{H}^{*}$ representing the identity in $H$ may be reduced to the identity by repeated deletion of subwords $r_{j} \in \mathcal{S}_{G_{C_{j}}}^{*}$ such that $r_{j}$ represents the identity in $G_{C_{j}}$. Proposition 4.8 describes an appropriately Lipschitz rectangular homotopy between words obtained by these deletions. Proposition 4.7 describes a part of the homotopy given in Proposition 4.8.

Given $v, w \in \mathcal{S}_{H}^{*}$, we will write $w=\mathcal{S}_{H}^{*} v$ if $v$ and $w$ are the same word and write $w={ }_{H} v$ if $v$ and $w$ represent the same element of $H$. Similar notation will be used for equality in other groups when there is any ambiguity. 
Proposition 4.6 Given a word $w \in \mathcal{S}_{H}^{*}$ such that $w={ }_{H} 1$, there exists a natural number $n \leq \ell(w)$ and, for $j=0, \ldots, n$, words $a_{j}, r_{j}, b_{j} \in \mathcal{S}_{H}^{*}$ with the following properties.

(i) $w=\mathcal{S}_{H}^{*} a_{0} r_{0} b_{0}$.

(ii) $a_{n}, r_{n}, b_{n}=\varepsilon$.

(iii) For all $j=0, \ldots, n$, there is some $C_{j} \in \mathcal{C}$ such that $r_{j} \in \mathcal{S}_{G_{C_{j}}}^{*}$ and $r_{j}={ }_{G_{C_{j}}}$.

(iv) $a_{j} b_{j}=\mathcal{S}_{H}^{*} a_{j+1} r_{j+1} b_{j+1}$ for $j=0, \ldots, n-1$.

Proof Note that each element of $\mathcal{S}_{H}$ lies in some $G_{C}$. If $w \neq \varepsilon$ represents the identity, then by the theory of free products, $w$ has a (nonempty) subword that is comprised of elements of some $\mathcal{S}_{G_{C}}$ and represents the identity in $G_{C}$. Thus, we may write $w=\mathcal{S}_{H}^{*}$ $a_{0} r_{0} b_{0}$, where $r_{0} \in \mathcal{S}_{G_{C_{0}}}^{*}$ as desired. Applying this argument recursively, we obtain $a_{j}, r_{j}, b_{j}$ as desired.

Proposition 4.7 Given a natural number $k$ and $w \in \mathcal{S}_{H}^{*}$, there exists an $O(k+\ell(w))$ Lipschitz map $f:[0,1] \times\left[0, \frac{k}{k+\ell(w)}\right] \rightarrow G$ with the following properties.

(i) Along the bottom, $f$ is given by $1^{k} w$, meaning $f(t, 0)=\gamma_{1^{k} w}(t)$, for $0 \leq t \leq 1$.

(ii) Along the top, $f$ is given by $w 1^{k}$, meaning $f\left(t, \frac{k}{k+\ell(w)}\right)=\gamma_{w 1^{k}}(t)$, for $0 \leq t \leq 1$.

(iii) $f$ is constant on the sides, so $f(0, s)$ and $f(1, s)$ do not depend on $s$.

Proof Let $\gamma: \mathbb{R} \rightarrow G$ be given as follows: $\gamma(t)=1_{G}$, for $t \leq 0, \gamma(t)=\gamma_{1^{k} w}(t)$, for $0<t \leq 1$, and $\gamma(t)=\gamma_{1^{k} w}(1)$, for $t>1$. Observe that $\operatorname{Lip}(\gamma)=O(k+\ell(w))$ Then we may take $f(t, s)=\gamma(t+s)$ as our desired filling.

Proposition 4.8 Given $a, b \in \mathcal{S}_{H}^{*}, r \in \mathcal{S}_{G_{C}}^{*}$ a relation in $G_{C}$ for some $C \in \mathcal{C}$, and any natural number $k$, let $\ell=\ell\left(\operatorname{arb} 1^{k}\right)$ and $h=\frac{\ell(r)}{\ell}$. There exists an $O(\ell)$-Lipschitz map $f:[0,1] \times[0, h] \rightarrow G$ with the following properties.

(i) Along the bottom, $f$ is given by arb $1^{k}$, meaning $f(t, 0)=\gamma_{a r b 1^{k}}(t)$, for $t \in[0,1]$.

(ii) Along the top, $f$ is given by $a b 1^{k+\ell(r)}$, meaning

$$
f(t, h)=\gamma_{a b 1^{k+\ell(r)}}(t), \quad \text { for } t \in[0,1] .
$$

(iii) $f$ is constant on the sides, so $f(0, s)$ and $f(1, s)$ do not depend on $s$.

Proof (See the right-hand side of Figure 3.) Subdivide the rectangle into $[0,1] \times$ $[0, h / 2]$ and $[0,1] \times[h / 2,1]$. Define $f$ to be $a 1^{\ell(r)} b 1^{k}$ on $[0,1] \times\{h / 2\}$, i.e., $f(t, h / 2)=$ $\gamma_{a 1^{e}(r) b 1^{k}}(t)$.

First, we extend $f$ over the top rectangle $[0,1] \times[h / 2,1]$. For

$$
(t, s) \in\left[0, \frac{\ell(a)}{\ell}\right] \times[h / 2,1] \cup\left[1-\frac{k}{\ell}, 1\right] \times[h / 2,1],
$$

we have that $\gamma_{a 1^{r} b 1^{k}}(t)=\gamma_{a b 1^{e(r)+k}}(t)$, so we can just set $f$ to be constant vertically, i.e., we define $f(t, s)=\gamma_{a b 1^{e}(r)+k}(t)$ for these $(t, s)$. To extend $f$ to

$$
(t, s) \in\left[\frac{\ell(a)}{\ell}, 1-\frac{k}{\ell}\right] \times[h / 2,1]
$$



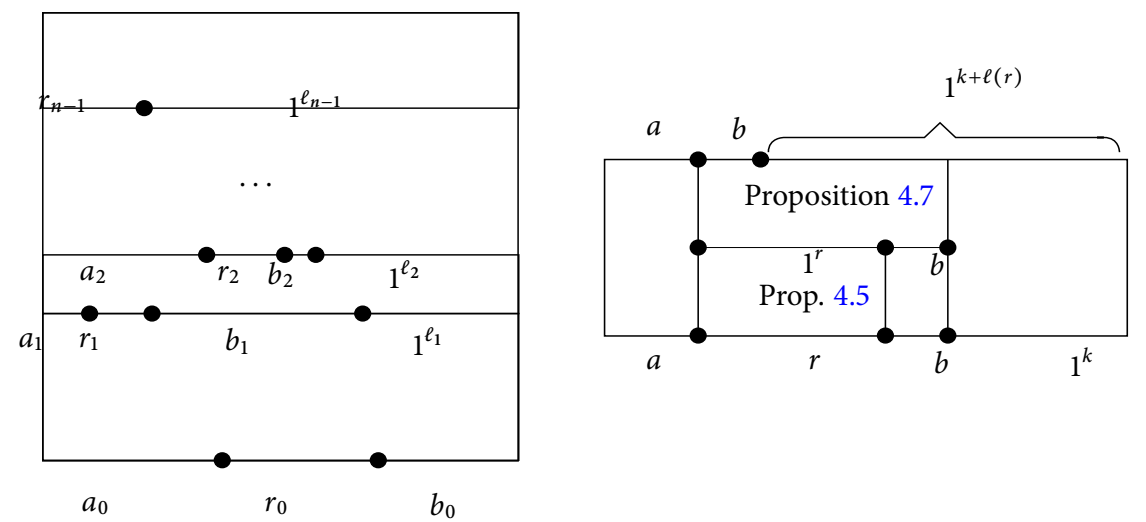

Figure 3: The figure on the left depicts our strategy for filling of the freely trivial word $w=$ $a_{0} r_{0} b_{0}$, where the $a_{j}, r_{j}, b_{j}$ are as in Proposition 4.6. The figure on the right depicts the proof of Proposition 4.8 that allows us to fill in each rectangle in the left-hand figure.

we simply apply Proposition 4.7. Thus, we have given an $O(\ell)$-Lipschitz extension of $f$ over the top rectangle.

Now we extend over the bottom rectangle $[0,1] \times[0, h / 2]$. For

$$
(t, s) \in\left[0, \frac{\ell(a)}{\ell}\right] \times[0, h / 2] \cup\left[\frac{\ell(a r)}{\ell}, 1\right] \times[0, h / 2],
$$

define $f(t, s)=\gamma_{a r b 1^{k}}(t)$. Finally, we apply Proposition 4.5 to extend $f$ over

$$
\left[\frac{\ell(a)}{\ell}, \frac{\ell(a r)}{\ell}\right] \times[0, h / 2]
$$

since this is equivalent to filling $r$.

Lemma 4.9 Recalling the notation introduced at the start of Section 4.7, we have $w \sim \mathcal{E}$ (in $G$ ) for all $w \in \mathcal{S}_{H}^{*}$ such that $w={ }_{H} 1$.

Proof (See Figure 3.) Let $w \in \mathcal{S}_{H}^{*}$ be a relation in $H$ and take a sequence of words $a_{j}, r_{j}, b_{j}, j=0, \ldots, n$ as in Proposition 4.6. We will define an $O(\ell(w))$-Lipschitz filling $f:[0,1] \times[0,1] \rightarrow G$ of $\gamma_{w}$ as follows. Let $\ell_{k}=\sum_{j<k} \ell\left(r_{j}\right)$, so that $\ell_{0}=0$ and $\ell_{n}=$ $\ell(w)$, and subdivide $[0,1] \times[0,1]$ into rectangles $[0,1] \times\left[\ell_{j}, \ell_{j+1}\right]$ for $j=0, \ldots, n-1$. Set $f\left(t, \ell_{j}\right)=\gamma_{a_{j} r_{j} b_{j} l^{\ell_{j}}}(t)$, noting that $\ell\left(a_{j} r_{j} b_{j} 1^{\ell_{j}}\right)=\ell(w)$.

Proposition 4.8 now shows that $f$ may be extended over each rectangle $[0,1] \times$ $\left[\ell_{j}, \ell_{j+1}\right]$ with Lipschitz constant $O(\ell(w))$.

\subsection{Generalizing Lemma 4.9.}

A careful examination of the proof of Lemma 4.9 shows that we have not used most of our hypotheses. In particular, the same proof shows that if $G_{1}, \ldots, G_{n}$ are compactly 
presented groups whose presentation complexes have Lipschitz 1-connected universal covers, then the presentation complex of the free product $G_{1} * \cdots * G_{n}$ has Lipschitz 1-connected universal cover as well.

\subsection{Distortion}

We now see that if $G$ is standard solvable, then elements of $U$ may be expressed much more efficiently in the generators of $G$ than in the generators of $U$.

Proposition 4.10 Suppose $G=U \rtimes A$ is standard solvable, $\mathcal{S}$ is a compact generating set for $G$, and $\mathcal{S}_{U}$ is a compact generating set for $U$. There exists $C>1$ such that if $u \in U \backslash\left\{1_{U}\right\}$, then $\frac{1}{C} \log \left(1+|u|_{\delta_{U}}\right) \leq|u|_{\delta} \leq C \log \left(1+|u|_{\delta_{U}}\right)$.

Proof This follows, with some effort, from [4, Proposition 6.B.2].

\subsection{The Multiamalgam}

In this subsection, we will define the multiamalgam $\widehat{G}$ of a standard solvable group $G=U \rtimes A$ (first introduced by Abels [1]), and quote a key theorem of Cornulier and Tessera that states certain conditions under which $G \cong \widehat{G}$. This means that $G$ is put together from its standard tame subgroups in a nice way, which will eventually let us build fillings in $G$ from fillings in standard tame subgroups. In order to state this theorem in the proper generality, we must briefly discuss the theory of unipotent groups.

\subsection{Unipotent Groups}

For a commutative $\mathbb{R}$-algebra $\mathcal{P}$, and a real unipotent group $U$, i.e., a closed group of upper triangular real matrices with diagonal entries equal to 1 , the theory of algebraic groups allows us to define a group $U(\mathcal{P})[2, \$ 1.4]$. In particular, if $U \subset \operatorname{GL}(n ; \mathbb{R})$ consists of all upper triangular matrices with diagonal entries equal to 1 , then $U(\mathcal{P})$ consists of all upper triangular $n \times n$ matrices over $\mathcal{P}$ with diagonal entries equal to 1 ; such matrices are certainly invertible, having determinant equal to 1 . Suppose $\mathcal{P}=$ $\mathbb{R}^{Y}$, so that $\mathcal{P}$ consists of all functions $f: Y \rightarrow \mathbb{R}$. Then there is an obvious bijection $U(\mathcal{P}) \leftrightarrow U^{Y}$, and for $y \in Y$ and $\widetilde{u} \in U(\mathcal{P})$ we may speak of $\widetilde{u}(y) \in U$.

Definition $4.11([4, \S 10 . \mathrm{B}]) \quad$ Let $G=U \rtimes A$ be real standard solvable. The multiamalgam $\widehat{G}$ of the standard tame subgroups $G_{C}$ is defined by $\widehat{G}=*_{C \in \mathcal{C}} G_{C} /\left\langle\left\langle R_{G}\right\rangle\right\rangle$, where $R_{G}=\left\{i_{C}(u)^{-1} i_{C^{\prime}}(u): u \in G_{C} \cap G_{C^{\prime}}\right\}$ and $i_{C}$ denotes the inclusion of $G_{C}$ in the direct product.

Similarly, the multiamalgam $\widehat{U}$ is defined by $\widehat{U}={ }{ }_{C \in \mathcal{C}} U_{C} /\left\langle\left\langle R_{U}\right\rangle\right\rangle$, where $R_{U}=$ $\left\{i_{C}(u)^{-1} i_{C^{\prime}}(u): u \in U_{C} \cap U_{C^{\prime}}\right\}$ and $i_{C}$ denotes the inclusion of $U_{C}$ in the direct product.

For any commutative $\mathbb{R}$-algebra $\mathcal{P}$, we define $\widehat{U}(\mathcal{P})$ and $\widehat{G}(\mathcal{P})$ similarly, where $G_{C}(\mathcal{P})$ is understood to be $U_{C}(\mathcal{P}) \rtimes A$. 
Of course, $\widehat{U} \rtimes A \cong \widehat{G}$. Recall that $G$ admits the SOL obstruction if it surjects onto a group of SOL type. Cornulier and Tessera give conditions under which $\widehat{U}$ is isomorphic to $U$.

Theorem 4.12 Let $G=U \rtimes A$ be a standard solvable real Lie group. If $H_{2}(\mathfrak{u})_{0}=0$, $\operatorname{Kill}_{2}(\mathfrak{u})_{0}=0$, and $G$ does not admit the SOL obstruction, then $\widehat{U}(\mathcal{P}) \cong U(\mathcal{P})$ for all commutative $\mathbb{R}$-algebras $\mathcal{P}$.

Proof This follows from [4, Corollary 9.D.4]. The 2-tameness hypotheses of the corollary is satisfied from [4, Proposition 4.C.3].

\section{Proof of the Main Theorem}

The rest of this paper is devoted to the proof of the following theorem.

Theorem 5.1 Let $G=U \rtimes A$, where $U$ and $A$ are contractible real Lie groups, $A$ is abelian, and $U$ is a real unipotent group, i.e., a closed group of strictly upper triangular real matrices. If $G$ is standard solvable and does not surject onto a group of SOL type, and $\mathrm{H}_{2}(\mathfrak{u})_{0}$ and $\mathrm{Kill}(\mathfrak{u})_{0}$ are trivial, then $\mathrm{G}$ is Lipschitz 1-connected.

Proof Lemma 5.2 will show that there exists a generating set $\mathcal{S}$ for $G$ and normal form $\omega: G \rightarrow \mathcal{S}^{*}$ with certain properties. Lemma 5.6 will show that if $\omega$ has these properties, then $\Delta \leadsto \varepsilon$ for $\omega$-triangles $\Delta$. By Lemma 3.8, this will suffice to prove the theorem.

\subsection{Defining $\omega$}

Assumption Throughout the rest of this paper, we assume that $G$ satisfies the hypotheses of the theorem. That is, $G=U \rtimes A$ is a standard solvable group such that $H_{2}(\mathfrak{u})_{0}=0$, $\mathrm{Kill}_{2}(\mathfrak{u})_{0}=0$, and $G$ does not surject onto a group of SOL type.

Notation. Let $H=\star_{C \in \mathcal{e}} G_{C}$ and $H_{U}[\mathcal{P}]=\star_{C \in \mathcal{C}} U_{C}(\mathcal{P})$ for any commutative $\mathbb{R}$ algebra $\mathcal{P}$, and let $i_{C}: U_{C}(\mathcal{P}) \rightarrow H_{U}[\mathcal{P}]$ denote inclusion. We will write $H_{U}$ for $H_{U}[\mathbb{R}]$. Let $\mathcal{S}_{A}$ be a compact generating set for $A$. For $C \in \mathcal{C}$, let $\mathcal{S}_{C}$ be a compact generating set for $U_{C}$. Let $\mathcal{S}_{U}=\bigcup_{C \in \mathcal{C}} \mathcal{S}_{C}$; by Theorem 4.12 this is a compact generating set for $U$. Let $\mathcal{S}=\mathcal{S}_{A} \cup \mathcal{S}_{U}$; this is a compact generating set for $G$. Let $\mathcal{S}_{H}$ be a generating set for $H$, which is equal to the union of compact generating sets for $G_{C}$ as $C$ ranges over $\mathcal{C}$, and let $\mathcal{S}_{H_{U}} \subset H_{U}$ be the union of the $\mathcal{S}_{C}$; this is a generating set for $H_{U}$. Given $C \in \mathcal{C}$ and $x \in U_{C}$, let $\bar{x} \in\left(\mathcal{S}_{A} \cup \mathcal{S}_{C}\right)^{*}$ be a minimal length word representing $x$. Let $\phi_{A}: G \rightarrow A$ be projection. Let the set theoretic map $\phi_{U}: G \rightarrow U$ be defined by $\phi_{U}(g)=\phi_{A}(g)^{-1} g$, so that $g=\phi_{A}(g) \phi_{U}(g)$.

Lemma 5.2 Under our standing assumptions, there exists a finite sequence $C_{1} \cdots C_{k}$ of conic subsets and a normal form $\omega: G \rightarrow \mathcal{S}^{*}$ such that $\omega$ has the following properties.

(i) For any $g \in G, \omega(g)=\omega\left(\phi_{A}(g)\right) \omega\left(\phi_{U}(g)\right)$.

(ii) For $a \in A, \omega(a) \in \mathcal{S}_{A}^{*}$ is a minimal length word representing $a$.

(iii) For $u \in U, \omega(u)$ has the form $\overline{x_{1}} \ldots \overline{x_{k}}$, where $x_{i} \in U_{C_{i}}$. 
Proof This follows from [4, Proposition 6.B.2], but we will now give a different proof in order to introduce a trick that will be used later.

\subsection{The Cornulier-Tessera Trick}

For a set $Y$, define the commutative $\mathbb{R}$-algebra $\mathcal{P}_{Y}$ as the collection of all functions $f: Y \times[1, \infty) \rightarrow \mathbb{R}$ such that there is some $\beta \in \mathbb{N}$ with $|f(y, t)|<(1+t)^{\beta}$. Note that an element of $U\left(\mathcal{P}_{Y}\right)$ can be identified with a function from $Y \times[1, \infty)$ to $U$. Alternatively, one can think of an element of $U\left(\mathcal{P}_{Y}\right)$ as a family of functions $[1, \infty) \rightarrow U$, indexed by $Y$ with matrix coefficients uniformly bounded by some polynomial $(1+t)^{\beta}$.

Choose $Y$ to have at least continuum cardinality, and let $\widetilde{g} \in U\left(\mathcal{P}_{Y}\right)$ be such that for every $g \in U$, there is some $y \in Y$ and $t=O\left(|g|_{S_{U}}\right)$ such that $\widetilde{g}(y, t)=g$. It is certainly possible to do this; for instance, one might take $Y=U$ and set $\widetilde{g}(y, t)$ to be $1_{U}$ for $t<|y|_{S_{U}}$ and $y$ for $t \geq|y|_{S_{U}}$. In claiming that $\widetilde{g} \in U\left(\mathcal{P}_{Y}\right)$, we have used the fact that the matrix coefficients of $g \in U$ are at most polynomial in $|g|_{S_{U}}$.

Since, by Theorem 4.12, $U\left(\mathcal{P}_{Y}\right)$ is generated by the union of the $U_{C}\left(\mathcal{P}_{Y}\right)$, we can write $\widetilde{g}=\widetilde{x}_{1} \cdots \widetilde{x}_{k}$, where each $\widetilde{x}_{i}$ is an element of some $U_{C_{i}}\left(\mathcal{P}_{Y}\right)$. Observe that there exist some $\alpha \in \mathbb{N}$ such that $\left|\widetilde{x}_{i}(y, t)\right|_{\delta_{c_{i}}} \leq(1+t)^{\alpha}$, for all $i=1, \ldots, k, y \in Y$, and $t \geq 1$.

Now let $g$ be an element of $U$. By definition of $\widetilde{g}$, there exist $y \in Y$ and $t=O\left(|g|_{S_{U}}\right)$ such that $\widetilde{g}(y, t)=g$. For $i=1, \ldots, k$, let $x_{i}=\widetilde{x}_{i}(y, t)$. We have that

$$
g=\widetilde{g}(y, t)=\widetilde{x}_{1}(y, t) \cdots \widetilde{x}_{k}(y, t)=x_{1} \cdots x_{k},
$$

and $x_{i} \in U_{C_{i}}\left(\mathcal{P}_{Y}\right)$ with $\left|x_{i}\right|_{\delta_{c_{i}}}=O\left(t^{\alpha}\right)=O\left(|g|_{\delta_{U}}^{\alpha}\right)$. Take $\omega(g)$ to be $\overline{x_{1}} \cdots \overline{x_{k}}$, and note that $|\omega(g)|_{\delta}=O\left(\log |g|_{\delta_{U}}\right)$ because $\left|\overline{x_{i}}\right|_{\delta_{A} \cup \delta_{C_{i}}}=O\left(\log \left|x_{i}\right|_{\delta_{C_{i}}}\right)=O\left(\log |g|_{\delta_{U}}\right)$ by Proposition 4.10 .

We have thus defined $\omega$ on elements of $U$, with the desired properties. Define $\omega$ on $A$ by taking $\omega(a)$ to be the shortest word in $\mathcal{S}_{A}^{*}$ representing $a \in A$. Extend $\omega$ to all of $g$ by setting $\omega(g)=\omega\left(\phi_{A}(g)\right) \omega\left(\phi_{U}(g)\right)$. We must show that $\omega$ is a normal form, i.e., that, for $g \in G, \ell(\omega(g))=O\left(|g|_{s}\right)$.

We have $\ell\left(\omega\left(\phi_{A}(g)\right)\right)=\left|\phi_{A}(g)\right|_{\delta}=O\left(|g|_{\delta}\right)$, so it suffices to show that

$$
\ell\left(\omega\left(\phi_{U}(g)\right)\right)=O\left(|g|_{S}\right) .
$$

If $w \in \mathcal{S}^{*}$ is a minimal length word representing some $g \in G$, note that $\omega\left(\phi_{A}(g)\right)^{-1} w$ represents $\phi_{U}(g)$. By Proposition 4.10, there is some constant $C>1$ not depending on $g$ such that $\left|\omega\left(\phi_{A}(g)\right)^{-1} w\right|_{\delta} \geq \frac{1}{C} \log \left|\phi_{U}(g)\right|_{\delta_{U}}$. Thus, since $\left|\omega\left(\phi_{U}(g)\right)\right|_{\delta}=$ $O\left(\log \left|\phi_{U}(g)\right|_{\delta_{U}}\right)$, we have that $\omega\left(\phi_{U}(g)\right)=O\left(\left|\phi_{A}(g)\right|_{\delta}+|w|_{\delta}\right)=O\left(|g|_{\mathcal{S}}\right)$ as desired.

\subsection{Filling $\omega$-triangles}

We wish to show that we can fill $\omega$ triangles, where $\omega$ is a normal form produced by Lemma 5.2. Proposition 5.5 will allow us to homotope $\omega$-triangles into relations of the form $\overline{x_{i}} \ldots \overline{x_{K}}$, where each $\overline{x_{i}}$ is a word in $\mathcal{S}_{A} \cup \mathcal{S}_{C_{i}}$, efficiently representing an element $x_{i}$ of $U_{C_{i}}$, where $C_{1}, \ldots, C_{K}$ is some fixed sequence of conic subsets. In order to fill such relations, recall from Theorem 4.12 that, under our standing assumptions, $U(\mathcal{P}) \cong \widehat{U}(\mathcal{P})$ for any commutative $\mathbb{R}$-algebra $\mathcal{P}$. Consequently, the kernel of $H_{U}[\mathcal{P}] \rightarrow U(\mathcal{P})$ is normally generated by elements of the form $i_{C}(u)^{-1} i_{C^{\prime}}(u)$, where 
$u \in U_{C}(\mathcal{P}) \cap U_{C^{\prime}}(\mathcal{P})$. In order to fill $\overline{x_{1}} \cdots \overline{x_{K}}$ in Corollary 5.4 , we will need to factor $x_{1} \cdots x_{K}$ in the free product $H_{U}$ as a product of a bounded number of elements of the form $g^{-1} i_{C}(u)^{-1} i_{C^{\prime}}(u) g$, where each $g \in H_{U}$ is a product of a bounded number of elements living in some factors $U_{C}$, with $|g|_{\mathcal{S}_{U}}$ and $|u|_{\mathcal{S}_{U}}$ controlled by some polynomial of $\sum_{j=1}^{K}\left|x_{j}\right| \mathcal{S}_{C_{j}}$.

Lemma 5.3 ([4, Lemma 7.B.1]) Suppose that our standing assumptions are satisfied. Given a sequence of conical subsets $C_{1}, \ldots, C_{K}$, there exist natural numbers $N, \mu, \beta$ such that, for any sequence $x_{i} \in U_{C_{i}}$ with $x_{1} x_{2} \cdots x_{K}={ }_{U} 1_{U}$, there is an equality of the form $x_{1} \cdots x_{K}={ }_{H_{U}}\left(g_{1} r_{1} g_{1}^{-1}\right) \cdots\left(g_{N} r_{N} g_{N}^{-1}\right)$ satisfying

(i) $g_{j}={ }_{H_{U}} g_{j 1} \cdots g_{j \mu}$, where $g_{j k} \in U_{C_{j k}}$ for some $C_{j k} \in \mathcal{C}$;

(ii) each $r_{j}$ is of the form $i_{C_{j}^{\prime}}\left(u_{j}\right) i_{C_{j}^{\prime \prime}}\left(u_{j}\right)^{-1}$ for some conical subsets $C_{j}^{\prime}, C_{j}^{\prime \prime}$ and some $u_{j} \in U_{C_{j}^{\prime}} \cap U_{C_{j}^{\prime \prime}}$

(iii) $\left|g_{j k}\right|_{\mathcal{S}_{C_{j k}}}=O\left(\ell^{\beta}\right),\left|u_{j}\right|_{\mathcal{S}_{C_{j}^{\prime}}}=O\left(\ell^{\beta}\right)$, and $\left|u_{j}\right|_{\mathcal{S}_{C_{j}^{\prime \prime}}}=O\left(\ell^{\beta}\right)$, where $\ell=1+\sum_{i=1}^{K}\left|x_{i}\right|$.

Proof This is a special case of [4, Lemma 7.B.1], but we will reprise most of the details here. We will use the same Cornulier and Tessera trick we used to prove Lemma 5.2. Take $\mathcal{P}_{Y}$ as in the proof of Lemma 5.2. Recall that $\tilde{x} \in U_{C}\left(\mathcal{P}_{Y}\right)$ may be thought of as a function from $Y \times[0, \infty)$ to $U_{C}$. Let $Y$ be a set with at least continuum cardinality, so that there exists $\left(\widetilde{x}_{1}, \ldots, \widetilde{x}_{K}\right) \in U_{C_{1}}\left(\mathcal{P}_{Y}\right) \times \cdots \times U_{C_{K}}\left(\mathcal{P}_{Y}\right)$ that has the following strong surjectivity property: for any $\left(x_{1} \cdots x_{K}\right) \in U_{C_{1}}\left(\mathcal{P}_{Y}\right) \times \cdots \times U_{C_{K}}\left(\mathcal{P}_{Y}\right)$ with $x_{1} \cdots x_{K}={ }_{U} 1$, there exists $y \in Y$ and $t=O\left(\left|x_{1}\right| \mathcal{S}_{C_{1}}+\cdots+\left|x_{K}\right| \mathcal{S}_{C_{K}}\right)$ with $\tilde{x}_{i}(y, t)=x_{i}$.

By Theorem 4.12, we know that there is some equality of the form

$$
\widetilde{x}_{1} \cdots \widetilde{x}_{K}=_{H_{U}\left[\mathcal{P}_{Y}\right]}\left(\widetilde{g}_{1}^{-1} \widetilde{r}_{1} \widetilde{g}_{1}\right) \cdots\left(\widetilde{g}_{N}^{-1} \widetilde{r}_{N} \widetilde{g}_{N}\right)
$$

where $\widetilde{g} \in * U_{C}\left(\mathcal{P}_{Y}\right)$ and each $\widetilde{r}_{j}$ has the form $i_{C_{j}^{\prime}}\left(\widetilde{u}_{j}\right)^{-1} i_{C_{j}^{\prime \prime}}\left(\widetilde{u}_{j}\right)$, for some conical subsets $C_{j}^{\prime}, C_{j}^{\prime \prime}$ and $\widetilde{u}_{j} \in U_{C_{j}^{\prime}}\left(\mathcal{P}_{Y}\right) \cap U_{C_{j}^{\prime \prime}}\left(\mathcal{P}_{Y}\right)$. Since the $U_{C}\left(\mathcal{P}_{Y}\right)$ generate $H_{U}\left[\mathcal{P}_{Y}\right]$, there must be some $\mu$ such that for all $j=1, \ldots, N, \widetilde{g}_{j}=\widetilde{g}_{j 1} \ldots \widetilde{g}_{j \mu}$, where each $\widetilde{g}_{j k}$ lives in $U_{C_{j k}}$ for some $C_{j k} \in \mathcal{C}$. Note that by definition of $\mathcal{P}_{Y}$, there is some $\beta$ such that all $\left|\widetilde{g}_{j k}(y, t)\right|_{\delta_{U}}$ and $\left|\widetilde{u}_{j}(y, t)\right|_{\mathcal{S}_{C_{j}^{\prime}}}$ are $O\left(t^{\beta}\right)$.

Given, $x_{1}, \ldots, x_{K} \in U_{C_{1}} \times \cdots \times U_{C_{K}}$, let $\ell=\sum_{i=1}^{K}\left|x_{i}\right|_{\mathcal{S}_{C_{i}}}$ and choose $y \in Y$ and $t=O(\ell)$ such that $\tilde{x}_{i}(y, t)=x_{i}$, for $i=1, \ldots, K$. For $j=1, \ldots, N$ and $k=1, \ldots, \mu$, let $g_{j}=\widetilde{g}_{j}(y, t), g_{j k}=\widetilde{g}_{j k}(y, t), u_{j}=\widetilde{u}_{j}(y, t)$, and $r_{j}=i_{C^{\prime}}\left(u_{j}\right)^{-1} i_{C^{\prime \prime}}\left(u_{j}\right)$. It follows that $x_{1} \cdots x_{K}=H_{U}\left(g_{1} r_{1} g_{1}^{-1}\right) \cdots\left(g_{N} r_{N} g_{N}^{-1}\right)$, and the $g_{j}$ and $r_{j}$ satisfy the desired conditions.

Corollary 5.4 Suppose that our standing assumptions are satisfied. Given a sequence of conical subsets $C_{1}, \ldots, C_{K}$, we have in $G$ that $\overline{x_{1}} \cdots \overline{x_{K}} \leadsto \varepsilon$. for any sequence $x_{i} \in U_{C_{i}}$ with $x_{1} x_{2} \cdots x_{K}={ }_{U} 1_{U}$.

Proof By Lemma 5.3, we have (for $\beta, N, \mu$ independent of the $x_{j}$ ),

$$
x_{1} \cdots x_{K}={ }_{H_{U}}\left(g_{1} r_{1} g_{1}^{-1}\right) \cdots\left(g_{N} r_{N} g_{N}^{-1}\right),
$$


where $g_{j}={ }_{H_{U}} g_{j 1} \cdots g_{j \mu}$ for $g_{j k} \in U_{C_{j k}}$, each $r_{j}$ is of the form $i_{C}^{\prime}\left(u_{j}\right)^{-1} i_{C^{\prime \prime}}\left(u_{j}\right)$ for some conical subsets $C_{j}^{\prime}, C_{j}^{\prime \prime}$, and some $u_{j} \in U_{C_{j}^{\prime}} \cap U_{C_{j}^{\prime \prime}}$, and $\left|g_{j k}\right|_{\delta_{C_{j k}}}\left|u_{j}\right|_{S_{C_{j}^{\prime}}}=O\left(\ell^{\beta}\right)$, where $\ell=1+\sum_{i=1}^{K}\left|x_{i}\right|$.

For each $j=1, \ldots, N$, let $\overline{g_{j}} \in \mathcal{S}^{*}$ be $\overline{g_{j 1}} \ldots \overline{g_{j \mu}}$. Let $\overline{r_{j}} \in \mathcal{S}^{*}$ be equal to $\left(\bar{u}_{j}^{\prime}\right)^{-1} \bar{u}_{j}^{\prime \prime}$, where $\bar{u}_{j}^{\prime} \in \mathcal{S}_{A} \cup \mathcal{S}_{C_{j}^{\prime}}$ is a minimal length word representing $u_{j}$ in $G_{C_{j}^{\prime}}$ and $\bar{u}_{j}^{\prime \prime} \in \mathcal{S}_{A} \cup \mathcal{S}_{C_{j}^{\prime \prime}}$ is a minimal length word representing $u_{j}$ in $G_{C_{j}^{\prime \prime}}$. This implies that $\overline{r_{j}}$ represents $r_{j}$ in $H$.

First we show that $\overline{r_{j}} \leadsto \varepsilon$. Note that $C_{j}^{\prime} \cap C_{j}^{\prime \prime}$ is itself a conic subset, so there is some $\overline{u_{j}} \in\left(\mathcal{S}_{A} \cup \mathcal{S}_{C_{j}^{\prime} \cap C_{j}^{\prime \prime}}\right)^{*}$ that represents $u_{j}$ in $G_{C_{j}^{\prime} \cap C_{j}^{\prime \prime}}$ with $\ell\left(\overline{u_{j}}\right)=O\left(\ell\left(\overline{r_{j}}\right)\right)$. Thus, by Proposition 4.5, we have $\overline{r_{j}}=\left(\bar{u}_{j}^{\prime}\right)^{-1} \bar{u}_{j}^{\prime \prime} \leadsto{\overline{u_{j}}}^{-1} \overline{u_{j}} \leadsto \varepsilon$.

Next, observe that

$$
\ell\left(\overline{g_{j}}\right)=\sum_{k=1}^{\mu} \ell\left(\overline{g_{j k}}\right)=\sum_{k=1}^{\mu} O\left(\log \left|g_{j k}\right|_{\delta_{c_{j k}}}\right)=O\left(\beta \log \left(1+\sum_{i=1}^{K}\left|x_{i}\right|\right)\right)=O\left(\ell\left(\overline{x_{1}} \cdots \overline{x_{K}}\right)\right),
$$

by Proposition 4.10, and $\ell\left(\overline{r_{i}}\right)=O\left(\ell\left(\overline{x_{1}} \cdots \overline{x_{K}}\right)\right)$ similarly.

Because

$$
\begin{gathered}
\overline{x_{1}} \cdots \overline{x_{K}}={ }_{H}\left({\overline{g_{1} r_{1} g_{1}}}^{-1}\right) \cdots\left({\overline{g_{N} r_{N} g_{N}}}^{-1}\right), \\
\ell\left(\left({\overline{g_{1} r_{1} g_{1}}}^{-1}\right) \cdots\left({\overline{g_{N} r_{N} g_{N}}}^{-1}\right)\right)=O\left(\ell\left(\overline{x_{1}} \cdots \overline{x_{K}}\right)\right),
\end{gathered}
$$

we have by Lemma 3.6 and Lemma 4.9 that

$$
\overline{x_{1}} \cdots \overline{x_{K}} \leadsto\left({\overline{g_{1} r_{1} g_{1}}}^{-1}\right) \cdots\left({\overline{g_{N} r_{N} g_{N}}}^{-1}\right) \leadsto\left({\overline{g_{1}}}_{\bar{g}_{1}}^{-1}\right) \cdots\left({\overline{g_{N}}}_{\bar{g}_{N}}^{-1}\right) \leadsto \varepsilon
$$

because $\overline{r_{j}} \leadsto \varepsilon$ as noted above.

We need one more proposition before we can fill $\omega$-triangles. Given $a, x \in G$, let ${ }^{a} x$ denote $a \times a^{-1}$.

Proposition 5.5 Fix a sequence of conical subsets $C_{1}, \ldots, C_{K} \in \mathcal{C}$. We have that $\omega(a) \overline{x_{1}} \ldots \overline{x_{K}} \leadsto \overline{{ }^{a} x_{1}} \ldots \overline{a_{x_{2}}} \omega(a)$ for all $a \in A$ and $x_{i} \in U_{C_{i}}$.

Proof By Proposition 4.5,

$$
\begin{aligned}
& \omega(a) \overline{x_{1}} \cdots \overline{x_{K}} \leadsto\left(\omega(a) \overline{x_{1}} \omega(a)^{-1}\right)\left(\omega(a) \overline{x_{2}} \omega(a)^{-1}\right) \cdots\left(\omega(a) \overline{x_{K}} \omega(a)^{-1}\right) \omega(a) \\
& \sim \overline{{ }^{a} x_{1}} \ldots \overline{{ }^{a} x_{K}} \omega(a),
\end{aligned}
$$

We now conclude the proof of our main theorem by showing that we can fill $\omega$ triangles.

Lemma 5.6 Under our standing assumptions, if $g_{1}, g_{2}, g_{3} \in G$ with $g_{1} g_{2} g_{3}{ }_{G} 1_{G}$, we have $\omega\left(g_{1}\right) \omega\left(g_{2}\right) \omega\left(g_{3}\right) \leadsto \varepsilon$.

Proof Recall that $\omega(g)$ has the form $\omega(a) \overline{x_{1}} \cdots \overline{x_{k}}$, where $\bar{u}_{i} \in \mathcal{S}_{A} \cup \mathcal{S}_{C_{i}}$ for some fixed sequence $C_{1}, \ldots, C_{k}$ of conical subsets. Let $g_{1}, g_{2}, g_{3} \in G$ with $g_{1} g_{2} g_{3}=1_{G}$, and let $a_{i}=\phi_{A}\left(g_{i}\right)$, for $i=1,2,3$. Let $\omega\left(\phi_{U}\left(g_{1}\right)\right)=\overline{x_{1}} \cdots \overline{x_{k}}, \omega\left(\phi_{U}\left(g_{2}\right)\right)=\overline{x_{1}^{\prime}} \cdots \overline{x_{k}^{\prime}}$ and 
$\omega\left(\phi_{U}\left(g_{3}\right)\right)=\overline{x_{1}^{\prime \prime}} \cdots \overline{x_{k}^{\prime \prime}}$. Expanding and applying Proposition 5.5 repeatedly, we slide the $a$-words to the right to see that

$$
\begin{aligned}
& \omega\left(g_{1}\right) \omega\left(g_{2}\right) \omega\left(g_{3}\right)=\omega\left(a_{1}\right) \overline{x_{1}} \cdots \overline{x_{k}} \omega\left(a_{2}\right) \overline{x_{1}^{\prime}} \cdots \overline{x_{k}^{\prime}} \omega\left(a_{3}\right) \overline{x_{1}^{\prime \prime}} \cdots \overline{x_{k}^{\prime \prime}} \\
& \leadsto \overline{a_{1} x_{1}} \ldots \overline{a_{1} x_{k}} \overline{a_{2} a_{1} x^{\prime}}{ }_{1} \ldots \overline{a_{2} a_{1} x_{k}^{\prime}} \overline{a_{3} a_{2} a_{1} x^{\prime \prime}}{ }_{1} \ldots \overline{a_{3} a_{2} a_{1} x_{k}^{\prime \prime}} \omega\left(a_{1}\right) \omega\left(a_{2}\right) \omega\left(a_{3}\right)
\end{aligned}
$$

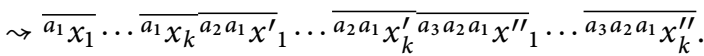

This resulting word admits a Lipschitz filling by Corollary 5.4.

Acknowledgments We wish to thank Robert Young and Yves Cornulier for productive conversations.

\section{References}

[1] Herbert Abels, Finite presentability of S-arithmetic groups. In: Compact presentability of solvable groups. Lecture Notes in Mathematics, 1261. Springer-Verlag, Berlin, 1985. http://dx.doi.org/10.1007/BFb0079708

[2] Armand Borel, Linear algebraic groups. Second edition. Graduate Texts in Mathematics, 126. Springer-Verlag, New York, 1991.

[3] Martin R. Bridson and André Haefliger, Metric spaces of non-positive curvature. Grundlehren der Mathematischen Wissenschaften, 319. Springer-Verlag, Berlin, 1999. http://dx.doi.org/10.1007/978-3-662-12494-9

[4] Yves Cornulier and Romain Tessera, Geometric presentations of Lie groups and their Dehn functions. Publ. Math. Inst. Hautes Études Sci. 125(2017), 79-219. http://dx.doi.org/10.1007/s10240-016-0087-3

[5] David Epstein, Mike S. Paterson, James W. Cannon, Derek F. Holt, Silvio V Levy, and William P Thurston, Word processing in groups. Jones and Bartlett, Boston, MA, 1992.

[6] Mikhail Gromov, Asymptotic invariants of infinite groups. In: Geometric group theory. Vol. 2, London Math. Soc. Lecture Notes Series, 182, Cambridge University Press, Cambridge, 1993.

[7] Shoshichi Kobayashi and Katsumi Nomizu, Foundations of differential geometry. Volume 1. Interscience Publishers, New York, 1963.

[8] Alexander Lytchak, Stefan Wenger, and Robert Young, Dehn functions and Hölder extensions in asymptotic cones. arxiv:1608.00082, 2016.

[9] Ayato Mitsuishi and Takao Yamaguchi, Locally Lipschitz contractibility of Alexandrov spaces and its applications. Pacific J. Math. 270(2014), no. 2, 393-421. http://dx.doi.org/10.2140/pjm.2014.270.393

[10] Robert Young, The Dehn function of SL( $n, Z)$. Ann. of Math. 177(2013), no. 3, 969-1027. http://dx.doi.org/10.4007/annals.2013.177.3.4

Department of Mathematics, University of Chicago, Chicago, IL 60637, USA

e-mail: davidbrucecohen@gmail.com 